\title{
Optimization-based load reduction during rapid shut-down of multi-megawatt wind turbine generators
}

\author{
Sébastien Gros $^{1}$ and Benoît Chachuat ${ }^{2}$ \\ ${ }^{1}$ Optimization in Engineering Center (OPTEC), K.U.Leuven, Kasteelpark Arenberg 10 postbus 2440, B-3001 Heverlee, Belgium \\ ${ }^{2}$ Centre for Process Systems Engineering (CPSE), Department of Chemical Engineering, Imperial College London, SW7 2AZ, UK
}

\begin{abstract}
This paper describes an optimization-based approach to reducing extreme structural loads during rapid or emergency shutdown of multi-megawatt wind turbine generators. The load reduction problem is cast into an optimal control formulation, and a simple, low-order model is developed in order for this optimization problem to be tractable in reasonable time using state-of-the-art numerical methods. To handle the variations in wind speed and turbulence inherent to wind turbine operation as well as the presence of model mismatch, a real-time optimization strategy based on fast sensitivity updates is also considered, whose on-line computational burden is limited to the repeated solution of quadratic programs that are designed off-line. The low-order model and both the open-loop and closed-loop optimal control strategies are validated against a high-fidelity model in the simulation environment Bladed ${ }^{\mathrm{TM}}$ for an industrial $3 \mathrm{MW}$ wind turbine. Under favorable shut-down scenarios, i.e. when the wind turbine is operating properly and the actuators and sensors are not faulty, large reductions of the first compressive peak and subsequent compressive/tensile peaks of the tower load pattern are obtained at various above-rated wind speeds compared to normal pitch control shutdown. Extension to more challenging shut-down scenarios are also discussed. Copyright (C) 2012 John Wiley \& Sons, Ltd.
\end{abstract}

KEYWORDS

wind turbine; rapid shut-down; emergency shut-down; fatigue load reduction; extreme loads; dynamic optimization; optimization-based control

Correspondence

E-mail: b.chachuateimperial.ac.uk

Received...

\section{INTRODUCTION}

With the size of wind turbine generators (WTG) steadily increasing, there has been a growing interest in alleviating structural fatigue through better control [1, 2, 3]. For multi-megawatt WTG (MMWTG) in particular, fatigue load on the tower has received special attention since the cost of the foundations can contribute a significant part of a WTG total cost, up to $40 \%$ for an off-shore wind turbine [4]. The control of tower fatigue during normal power production operation has mainly focused on the tower fore-aft oscillations that have a direct impact on the WTG foundations. Such oscillations are commonly reduced through collective pitch control, using linear feedback based on the measurements of the WTG nacelle fore-aft acceleration. More recently, attention has shifted toward reducing the rotor imbalance through individual pitch control, using linear feedback based on non-linear (static) transformations of the blades load measurements and nacelle acceleration; see, e.g., [5, 6, 7].

Transient operation such as shut-down, on the other hand, has received little attention so far. Apart from normal, scheduled shut-downs, a number of hazardous situations require shutting-down the WTG as fast as possible to prevent damages and bring it to a safe state [8]. This includes shut-down due to high wind-speeds as well as rapid shut-down triggered by the loss of generator torque or pitch control, faulty sensors and actuators, or hazardous conditions such as blade icing. We shall refer to these shutdowns collectively as emergency shut-downs (EST) thereafter.

With no more than a few rapid shutdown events triggered every day-for instance due to high winds, see Figure 1 -the average fatigue loads incurred by EST events remain small in comparison to cumulative fatigue loads over the lifetime of 
a typical WTG. Despite being of short duration — a few seconds only, it is the magnitude of the loads experienced during these EST events which causes concern, for instance by impairing the WTG foundations or its structural integrity [8]. As just one recent example, the Boston Herald reported in its 25 February, 2012 edition* that a wind turbine in Charlestown, MA, was shut down for foundation fix after discovering that it had settled abount 2 inches, with vibrations from a sudden shutdown triggered by high winds cited as a likely cause. The fact that EST events can cause extreme tower loads has also been observed and studied in numerical simulation [9]. For instance, loss of generator torque coincident with a wind gust is classically a design-driving load case [10].

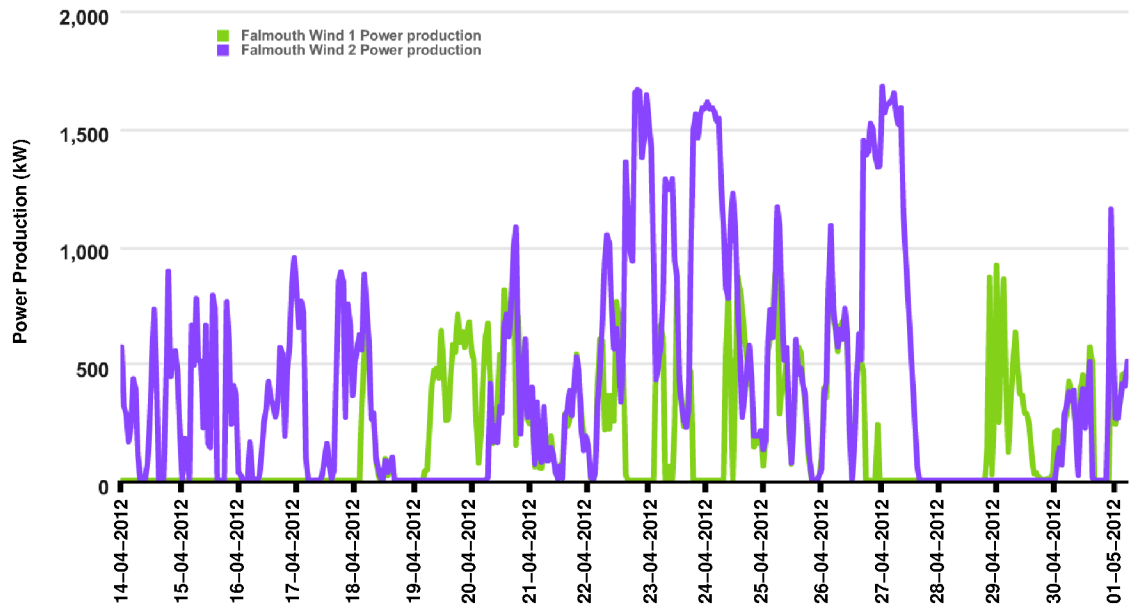

Figure 1. Power production of two $2 \mathrm{MW}$ wind turbines in Falmouth, MA, over the period 15 April, 2012 to 30 April, 2012. Source: http://www. powerdash.com/systems/1000475.

EST procedures commonly rely on a set of ad hoc rules, whereby the collective pitch control is ramped to feather, while the generator torque is either maintained at its maximum allowable value or set to zero [11]. In practice, a higher pitch rate can reduce the maximum positive load experienced for instance by the blades, but the resulting load of thrust then causes the tower top to swing upwind, resulting in a negative load peak which is typically greater in magnitude than the maximum positive load. Bossanyi et al. [9] argue that different pitch rates should be used during a rapid shutdown, a high pitch rate for long enough to limit the peak overspeed, followed by a lower rate to avoid the high negative loads. To accomplish this task, they also suggest that a model-based control strategy that would adjust the pitch rate dynamically to optimize the shutdown in a defined mathematical sense, knowing the turbine dynamics in detail. It is precisely the objective of this paper to investigate such an optimization-based methodology for extreme load reduction during EST events. It is also a principal aim to provide a proof-of-concept of this technology via validation against a high-fidelity model in the simulation environment Bladed $^{\mathrm{TM}}$. Note that the scope is mainly limited to the case that the WTG is operating properly and that the actuators and sensors are not faulty, although more challenging shut-down scenarios are discussed towards the end of the paper.

Reducing structural loads during EST events is challenging because good reference trajectories for the collective pitch rate and, to a lesser extent, for the generator torque are hard to assess in general. Moreover, the dynamics are fast and highly nonlinear during such events, the actuators are likely to become saturated, and other process constraints may become active too. In this context, linear control theory fails to provide an adequate framework for dealing with EST procedures. Problems with implicit control objectives, constraints, nonlinear dynamics, and multiple control variables are more naturally addressed under the framework of optimal control [12]. In particular, direct solution methods, whereby the optimal control problem (OCP) is converted into a nonlinear program (NLP) through parameterization of the control and/or state trajectories (e.g., using polynomial functions) can efficiently tackle OCPs with both point and path constraints as well as switched dynamics $[13,14,15,16]$. This is the approach taken in this work.

Because EST procedures are subject to large disturbances, such as variation in wind speed and turbulence, and since the mathematical models carry their load of uncertainty, direct application of the computed optimal control trajectories in open loop can lead to poor performance and may even violate some operating constraints. Pivotal to the success of an optimal control methodology for EST are closed-loop control strategies that adapt the control trajectories in real time by

* Source: http://www.windaction.org/news/34427. Industrial Wind Action is an independent group providing information on industrial wind energy based on the latest IWA announcements, news articles, and fact-based documents pertaining to wind energy development. 
accounting for the available on-line measurements [17, 18]. A number of dynamic real-time optimization (DRTO) methods have been developed in recent years to tackle such problems. One approach involves solving the OCP repeatedly-either in an exact way or in an approximate way using linearization - after incorporating the latest process information, for which advanced techniques have been devised that reduce the computational burden significantly [19, 20, 21, 22, 23]. Part of the computational burden can also be moved from on-line to off-line execution based on parametric programming techniques $[24,25]$, but to date this approach is mainly limited to linear dynamic systems.

The remainder of the paper is organized as follows. A low-order model is first developed in Section 2, which is well suited for rigorous optimization of EST procedures. The problem of reducing extreme loads during an EST event is formulated as an optimal control problem in Section 3 based on the low-order model, and the results of a case study are presented and analyzed in Section 4, including a validation in the high-fidelity simulation environment Bladed ${ }^{\mathrm{TM}}$. A computationally tractable, real-time optimization approach is then proposed in Section 5 for implementation of the optimal EST procedure in the presence of uncertainty and this approach is demonstrated on a case study. Section 6 discusses the application of the optimization-based methodology to more challenging shut-down scenarios. Finally, conclusions are drawn and directions for future investigations are identified in Section 7.

\section{LOW-ORDER MODEL FOR OPTIMIZATION OF EST PROCEDURES}

Several WTG high-fidelity models have been studied in the literature to date and a number of commercial simulators are available. A state-of-the-art and well accepted model is the one developed by GL Garrad Hassan, which is implemented in the simulation environment Bladed ${ }^{\mathrm{TM}}$ [26]. This model takes a large number of structural modes into account and has been proved to be highly accurate for a wide variety of WTGs, yet its high complexity proscribes its use within a systematic optimization procedure based on mathematical programming. In order to make the optimization of EST procedures tractable, a smooth and low-complexity model is needed. One such model is developed subsequently in Subsection 2.1, which accounts for the first fore-aft tower mode only. Its validity is discussed and illustrated by a comparison with the Bladed model for a 3 MW WTG in Subsection 2.2.

\subsection{Dynamic Model Formulation}

A schematic diagram of the WTG, with an indication of the state variables, is shown in Figure 2. $\Phi_{T}[\mathrm{rad}]$ stands for the tower fore-aft displacement relative to the vertical axis; $\Omega_{H}[\mathrm{rad} / \mathrm{s}]$, for the rotor angular velocity; and $\beta$ [deg], for the collective pitch position.

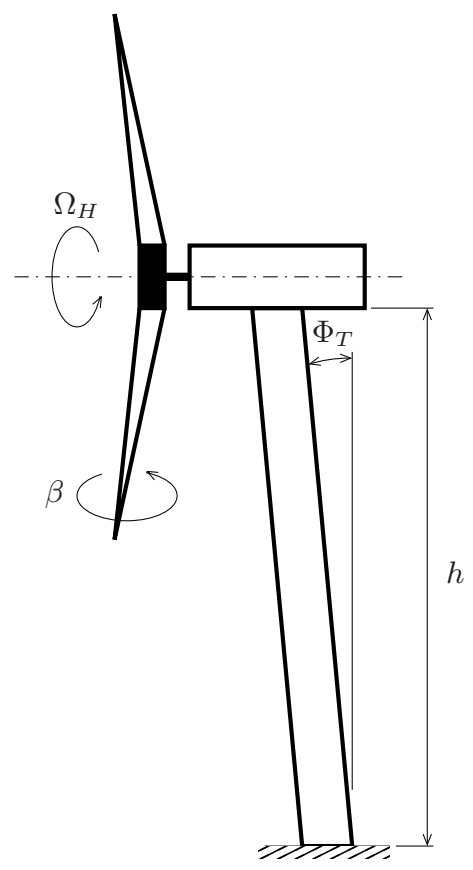

Figure 2. Schematic diagram of wind turbine generator. 
Dynamics A low-order model is developed through the Lagrange formalism. The Lagrangian for the WTG in Figure 2 is given by

$$
\mathcal{L}=\frac{1}{2}\left[\left(I_{D T}+J\right)\left(\Omega_{H}\right)^{2}+\left(J_{t}+M_{b l} h^{2}\right)\left(\xi \dot{\Phi}_{T}\right)^{2}-M_{r n}\left(\xi h w_{t} \Phi_{T}\right)^{2}\right],
$$

where a description of the model parameters can be found in Table I. The Lagrange equations for $\Phi_{T}$ and $\Omega_{H}$ read

$$
\begin{aligned}
\dot{\Omega}_{H} & =\frac{Q_{a}-T_{G}}{I_{D T}+J} \\
\ddot{\Phi}_{T} & =\frac{Q_{t}-\xi^{2} M_{r n} h^{2} w_{t}^{2} \Phi_{T}-Q_{m}}{\xi^{2}\left(M_{b l} h^{2}+J_{t}\right)}
\end{aligned}
$$

where $T_{G}[\mathrm{Nm}]$ stands for the generator torque. The rotor aerodynamic torque $Q_{a}[\mathrm{Nm}]$ (around the rotor axis), the thrust moment $Q_{t}[\mathrm{Nm}]$ (around the tower base axis) and the structural moment $Q_{m}[\mathrm{Nm}]$ in (2)-(3) are given by

$$
\begin{aligned}
Q_{a} & =\frac{1}{2} \rho \pi R^{3} C_{p \lambda} V^{2} \\
Q_{t} & =\frac{1}{2}\left(1+C_{s}\right) h \rho \pi R^{2} C_{t} V^{2}+C_{X} V^{2} \\
Q_{m} & =Q_{o v}+B_{t} \dot{\Phi}_{T},
\end{aligned}
$$

where $C_{s}$ is the parameter accounting for the vertical shift of the thrust caused by the wind shear ; $C_{t}$, the aerodynamic coefficient relative to the thrust; $C_{X}$, the aerodynamic coefficient relative to the structure drag; $C_{p \lambda}:=\frac{C_{p}}{\lambda}$, the aerodynamic coefficient relative to the rotor torque $\left(C_{p}\right.$ being the rotor efficiency coefficient); ${ }^{\dagger} Q_{o v}$, the mechanical moment yielded by the horizontal shift (along the wind direction axis) of the nacelle center of mass; and $B_{t}$, the tower structural damping factor. In addition, $\lambda[-]$ denotes the tip-speed ratio, which is calculated as

$$
\begin{aligned}
\lambda & =\frac{R \Omega_{H}}{V} \\
V & =V_{\infty}-\xi h \dot{\Phi}_{T},
\end{aligned}
$$

with $V[\mathrm{~m} / \mathrm{s}]$ the wind speed at the hub position, including the relative wind due to the tower motion, and $V_{\infty}[\mathrm{m} / \mathrm{s}]$ the free-flow wind speed. The aerodynamic coefficients are defined in look-up tables $C_{p \lambda}(\beta, \lambda)$ and $C_{t}(\beta, \lambda)$, as obtained from combined blade element and momentum (BEM) theory. BEM is a standard approach to characterizing the aerodynamic behavior of the rotor based on its design and the two-dimensional lift and drag coefficients for the blade profiles (typically computed by a blades' manufacturer using high-fidelity airfoil aerodynamic models), assuming no aerodynamic interaction between the blade elements. Besides the aerodynamic coefficients, the parameter $C_{s}$ accounting for the effect of the windshear on the turbine deflection typically varies with the average wind speed. Its value is fixed here as a first approximation.

Regarding the collective pitch, the actuators dynamics relating $\beta$ to the collective pitch reference $\beta_{R}[\mathrm{deg}]$ are approximated as second-order dynamics with a limited bandwidth,

$$
\ddot{\beta}=K_{P}\left(\beta_{R}-\beta\right)-K_{D} \dot{\beta} .
$$

Saturations are also taken into account for the pitch rate $\dot{\beta}$ and the pitch acceleration $\ddot{\beta}$ later on in Subsection 3.2.

Initial conditions The WTG is assumed to be operating in power production mode when an EST procedure is triggered. During such "normal" operation, the rotor velocity $\Omega_{H}^{\mathrm{r}}$, generator torque $T_{G}^{\mathrm{r}}$ and pitch angle $\beta^{\mathrm{r}}$ are usually determined to meet either one of two goals:

(i) maximize power production when the wind speed is too low for the WTG to reach its rated power value (below-rated condition);

(ii) limit power production at the rated power value, otherwise (above-rated condition).

The potential energy that is stored in the WTG structure increases with the wind speed when operated under below-rated conditions, and reaches a maximum at the transition between below-rated and above-rated conditions. It then stays around this maximum value for larger (above-rated) wind speeds. The tower fore-aft displacement, and hence the fatigue, during an ensuing EST is typically larger when triggered in above-rated conditions, which is the focus of this paper. Nevertheless,

${ }^{\dagger}$ The main reason for implementing $\frac{C_{p}}{\lambda}$ as a new variable $C_{p \lambda}$ is to avoid numerical issues when $C_{p} \rightarrow 0$ and $\lambda \rightarrow 0$. 
Table I. Low-order model parameters

\begin{tabular}{crll}
\hline Parameter & \multicolumn{2}{c}{ Value (Case Study) } & Description \\
\hline$J$ & $1.1366 \times 10^{7}$ & {$\left[\mathrm{~kg} \mathrm{~m}^{2}\right]$} & Rotor inertia \\
$J_{t}$ & $1.1810 \times 10^{9}$ & {$\left[\mathrm{~kg} \mathrm{~m}^{2}\right]$} & Tower fore-aft inertia \\
$I_{D T}$ & $3.541 \times 10^{6}$ & {$\left[\mathrm{~kg} \mathrm{~m}^{2}\right]$} & Drive-train inertia \\
$B_{t}$ & $4.8186 \times 10^{7}$ & {$[\mathrm{Nm} \mathrm{s} / \mathrm{rad}]$} & Tower fore-aft damping \\
$M_{r n}$ & 151490 & {$[\mathrm{~kg}]$} & Mass of the rotor and nacelle \\
$M_{b l}$ & 34791 & {$[\mathrm{~kg}]$} & Mass of the three blades \\
$R$ & 50.3 & {$[\mathrm{~m}]$} & Rotor radius \\
$h$ & 100.6 & {$[\mathrm{~m}]$} & Tower height \\
$\xi$ & 1.5 & {$[-]$} & Shape factor \\
$C_{s}$ & 0.1363 & {$[-]$} & Wind shear factor \\
$C_{X}$ & $1.2801 \times 10^{4}$ & {$\left[\mathrm{~N} / \mathrm{s}^{2}\right]$} & Tower and nacelle aerodynamic drag coefficient \\
$Q_{o v}$ & $1.0680 \times 10^{7}$ & {$[\mathrm{Nm}]$} & Overhang torque \\
$\rho$ & 1.225 & {$\left[\mathrm{~kg} / \mathrm{m}^{3}\right]$} & Density of air \\
$w_{t}$ & 2.55 & {$[\mathrm{rad} / \mathrm{s}]$} & Tower first-mode frequency \\
$K_{P}$ & 100 & {$\left[1 / \mathrm{s}^{2}\right]$} & Pitch actuators loop proportional gain \\
$K_{D}$ & 20 & {$[1 / \mathrm{s}]$} & Pitch actuators loop derivative gain \\
$T_{G}^{\mathrm{r}}$ & $2.15 \times 10^{6}$ & {$[\mathrm{Nm}]$} & Rated generator torque \\
$\Omega_{H}^{\mathrm{r}}$ & 15 & {$[\mathrm{rpm}]$} & Rated rotor velocity \\
\hline
\end{tabular}

the proposed optimization-based methodology can be applied to below-rated conditions subject to minor modifications of the initial conditions given subsequently. Attention is drawn to the fact that dynamic inflow can become a significant phenomenon in below-rated conditions and that the $C_{p \lambda}(\beta, \lambda)$ and $C_{t}(\beta, \lambda)$ curve approach may no longer be sufficient under these conditions though.

Under above-rated conditions, the rotor velocity $\Omega_{H}^{\mathrm{r}}$ is dictated by the WTG design requirements, while the generator torque $T_{G}^{\mathrm{r}}$ is adjusted to meet the rated power value. In particular, this requires that a constant rotor velocity is maintained by pitching the blades so as to control the aerodynamic torque. For a given wind speed $V_{\infty}$, the initial pitch $\beta(0)=\beta^{\mathrm{r}}$ is thus implicitly specified via (2) as

$$
0=\dot{\Omega}_{H}(0)=\frac{\frac{1}{2} \rho \pi R^{3} C_{p \lambda}\left(\beta^{\mathrm{r}}, \frac{R \Omega_{H}^{\mathrm{r}}}{V_{\infty}}\right) V_{\infty}^{2}-T_{G}^{\mathrm{r}}}{I_{D T}+J} .
$$

The other initial conditions can be specified as $\dot{\beta}(0)=0$ and $\ddot{\beta}(0)=0$ for the collective pitch, and $\dot{\Phi}_{T}(0)=0$ and $\ddot{\Phi}_{T}(0)=0$ regarding the tower displacement. In turn, these specifications constrain the initial tower displacement and pitch reference, respectively, via (3) and (4) as

$$
\begin{aligned}
\Phi_{T}(0) & =\frac{\frac{1}{2}\left(1+C_{s}\right) h \rho \pi R^{2} C_{t}\left(\beta^{\mathrm{r}}, \frac{R \Omega_{H}^{\mathrm{r}}}{V_{\infty}}\right) V_{\infty}^{2}+C_{X} V_{\infty}^{2}-Q_{o v}}{\xi^{2} M_{r n} h^{2} w_{t}^{2}} \\
\beta_{R}(0) & =\beta^{\mathrm{r}} .
\end{aligned}
$$


Model Summary The complete low-order model is given by

$$
\begin{array}{rlrl}
\dot{\Omega}_{H}(t) & =\frac{Q_{a}(t)-T_{G}(t)}{I_{D T}+J}, & & Q_{a}(0)=T_{G}^{\mathrm{r}}, \quad \Omega_{H}(0)=\Omega_{H}^{\mathrm{r}} \\
\ddot{\Phi}_{T}(t) & =\frac{Q_{t}(t)-\xi^{2} M_{r n} h^{2} w_{t}^{2} \Phi_{T}(t)-Q_{m}(t)}{\xi^{2}\left(M_{b l} h^{2}+J_{t}\right)}, & \dot{\Phi}_{T}(0)=0, & \ddot{\Phi}_{T}(0)=0 \\
\ddot{\beta}(t) & =K_{P}\left(\beta_{R}(t)-\beta(t)\right)-K_{D} \dot{\beta}(t), & \dot{\beta}(0)=0, & \ddot{\beta}(0)=0 \\
Q_{a}(t) & =\frac{1}{2} \rho \pi R^{3} C_{p \lambda}(\beta(t), \lambda(t)) V(t)^{2} & \\
Q_{t}(t) & =\frac{1}{2}\left(1+C_{s}\right) h \rho R^{2} C_{t}(\beta(t), \lambda(t)) V(t)^{2}+C_{X} V(t)^{2} & \\
Q_{m}(t) & =Q_{o v}+B_{t} \dot{\Phi}_{T}(t) & \\
\lambda(t) & =\frac{R \Omega_{H}(t)}{V(t)} & & \\
V(t) & =V_{\infty}-\xi h \dot{\Phi}_{T}(t) . &
\end{array}
$$

The controls in this model are $T_{G}(t)$ and $\beta_{R}(t)$. Observe that (7)-(14) is a fifth-order model, yet as many as six initial conditions are defined; that is, the system is over-specified at initial time, which implies that the initial control value $T_{G}(0)$ can be set freely, but the other initial control value $\beta_{R}(0)$ is imposed by (5) and (6).

By construction, the dynamic model (7)-(14) only takes into account the first fore-aft tower mode, while subsequent modes are neglected. The out-of-plane blade modes are also neglected, corresponding to the three blades oscillating in the fore-aft direction. Therefore, (7)-(14) may only predict the behavior of WTGs accurately in the lower frequency range, below the influence of the neglected modes. A comparison between the reduced model predictions and those of the commercial simulator Bladed is presented next to illustrate these limitations.

\subsection{Case Study}

A $3 \mathrm{MW}$ is considered as a case study throughout the paper, ${ }^{\ddagger}$ for which a Bladed model is available for detailed performance and loading simulation. Regarding the low-order model (7)-(14), the parameter values are those reported in Table I. Parameters such as masses, inertia, and WTG geometry are readily deduced from the WTG design; resonance frequencies are obtained from the Bladed model using modal analysis; the look-up tables $C_{p \lambda}(\beta, \lambda)$ and $C_{t}(\beta, \lambda)$ shown in Figure 3 are obtained from the blade element and momentum theory algorithm implemented in Bladed [26]; and the remaining parameter values are obtained by fitting both the models steady-state values and linearized dynamics.
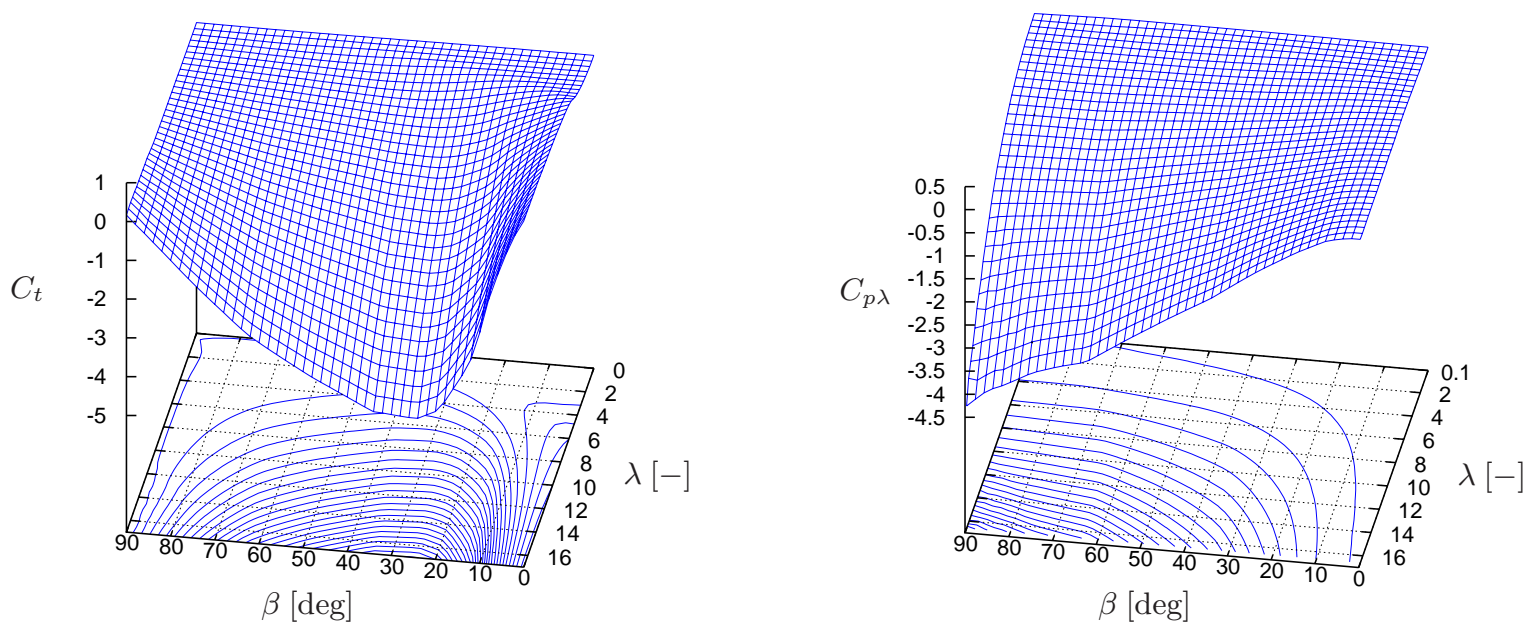

Figure 3. Aerodynamic coefficients $C_{t}$ (left plot) and $C_{p \lambda}$ (right plot) in the WTG case study.

${ }^{\ddagger}$ For confidentiality reasons, the parameters of an existing wind turbine could not be used directly. Therefore, the model proposed in this case study does not correspond to an existing WTG, although its behavior is representative of WTGs of similar sizes. 
A comparison between the linearized dynamics of the Bladed model with the linearized dynamics of the low-order model (7)-(14) is presented in Figure 4, for a free-flow wind speed of $V_{\infty}=22 \mathrm{~m} / \mathrm{s}$. The plots on the left-hand side give the magnitude and phase of the pitch-demand-to-tower-head-acceleration transfer function; those on the right-hand side give the magnitude and phase of the generator-torque-to-tower-head-acceleration transfer function.

The first peak around $2.5 \mathrm{rad} / \mathrm{s}$ corresponds to the first fore-aft tower mode and, as expected, the reduced model provides an excellent approximation in the lower frequency range $(<3 \mathrm{rad} / \mathrm{s})$. Above $3 \mathrm{rad} / \mathrm{s}$, however, the magnitudes and phases of both transfer functions appear to be diverging. It is found that the magnitude and phase at the right of the first fore-aft tower mode, between 3 and $5 \mathrm{rad} / \mathrm{s}$, are significantly influenced by the first out-of-plane blades mode. Then, the second peak in the Bladed transfer functions, around $11 \mathrm{rad} / \mathrm{s}$, corresponds to the second fore-aft tower mode. Similar model agreement is obtained at different free-flow wind speed values $V_{\infty}$.
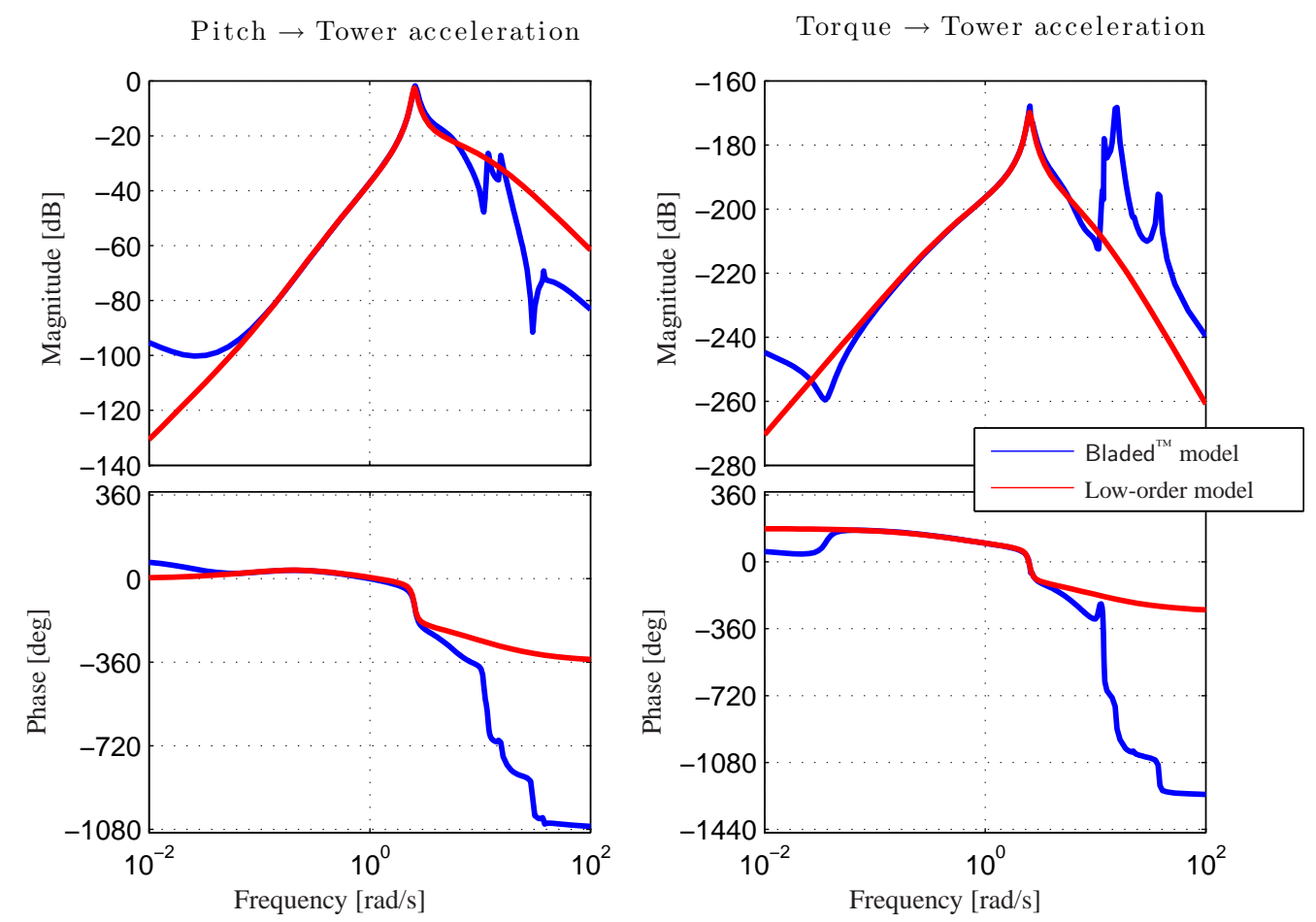

Figure 4. Pitch and generator torque to tower acceleration transfer functions at $V_{\infty}=22 \mathrm{~m} / \mathrm{s}$. Blue solid lines: transfer functions corresponding to the Bladed model; Red solid lines: transfer functions corresponding to the low-order model (7)-(14).

Further (indirect) validation of the low-order model (7)-(14) will be obtained later on in Subsection 4.3, when implementing the optimal shut-down procedures determined from that model in the Bladed model.

\section{OPTIMIZATION FORMULATION AND NUMERICAL SOLUTION}

This Section presents an approach to casting the problem of reducing the extreme loads during an EST procedure as an optimal control problem, based on the low-order model developed in Section 2. The formulation of a tractable objective function is discussed in Subsection 3.1, and operational constraints are described in Subsection 3.2. The complete optimal control problem and its numerical solution are discussed in Subsection 3.3.

\subsection{Structural Load Objective}

The objective of the optimization problem is to minimize the extreme loads during an EST procedure. Structural fatigue has been studied extensively in the literature and a well-accepted estimation technique is known as the rain-flow counting method (RFC) [27, 28, 11]. Simply put, RFC amounts to summing the peak-to-peak distances in a structural load pattern, 
such as the one shown in Figure 15, and is therefore well suited for minimizing extreme loads. A simple criterion approximating the structural load predicted by RFC over the finite shut-down horizon $\left[0, t_{\mathrm{f}}\right]$, as derived in Appendix A, is given by

$$
D=\int_{0}^{t_{\mathrm{f}}}\left|\dot{\Phi}_{T}(t)\right| d t
$$

The absolute value term in (15) can be numerically smoothened out in order to make the cost functional more convenient in a numerical optimization procedure, giving

$$
J=\int_{0}^{t_{\mathrm{f}}} \frac{\dot{\Phi}_{T}(t)^{2}}{\sqrt{\dot{\Phi}_{T}(t)^{2}+\epsilon^{2}}} d t
$$

with $\epsilon \ll 1$ a small numerical perturbation. It is also possible to give more weight to larger loads by considering higher powers of the integrand $\dot{\Phi}_{T}(t)$, for instance as $J=\int_{0}^{t_{\mathrm{f}}} \dot{\Phi}_{T}(t)^{2 n} d t$ with $n \geq 1$, or via the use of a min-max formulation, such as penalizing $\sup _{t \in\left[0, t_{\mathrm{f}}\right]}\left|\Phi_{T}\right|$ in the objective function [29].

\subsection{Operational Constraints}

The following path constraints are defined to represent the limitations imposed by the actuators on the pitch position, pitch rate and pitch acceleration,

$$
\beta^{\mathrm{br}} \leq \beta(t) \leq 90[\mathrm{deg}], \quad-\dot{\beta}^{\max } \leq \dot{\beta}(t) \leq \dot{\beta}^{\max }, \quad-\ddot{\beta}^{\max } \leq \ddot{\beta}(t) \leq \ddot{\beta}^{\max } .
$$

Moreover, a terminal constraint is defined in order for the pitch angles to be near to the feathered position at the final time $t_{\mathrm{f}}$ as

$$
\beta\left(t_{\mathrm{f}}\right) \geq \beta_{F}
$$

Note that the pitch angle $\beta(t)$ will only reach the feathered position (90 deg) asymptotically. The feathered angle is thus set as $\beta_{F}<90 \mathrm{deg}$ in order for the control problem to be feasible.

The generator torque $T_{G}$ and power $P_{G}:=T_{G} \Omega_{H}$ are restricted along the path as

$$
\begin{aligned}
0 \leq T_{G}(t) & \leq T_{G}^{\max }, \\
T_{G}(t) \Omega_{H}(t) & \leq 1.1 T_{G}^{\mathrm{r}} \Omega_{H}^{\mathrm{r}} .
\end{aligned}
$$

Finally, the maximum torque that can be reclaimed from the generator typically decreases with the rotor speed due to power electronics restrictions, and no torque can be reclaimed below the so-called cut-in rotor speed $\Omega^{\mathrm{ci}}$. A first, conservative approximation is considered subsequently of the form

$$
T_{G}(t) \leq \begin{cases}a \Omega_{H}(t)\left[\Omega_{H}(t)+b\right], & \text { if } \Omega_{H}(t) \geq \Omega^{\mathrm{ci}} \\ 0, & \text { otherwise, }\end{cases}
$$

where the constants $a$ and $b$ are selected in agreement with the power electronics characteristics of the WTG. The path constraints (19)-(21) are illustrated in Figure 5 for the parameter values given in Tables I and II. Note that the feasible domain for $T_{G}$ is the union of the shaded region with the line $T_{G}=0$, which clearly yields a nonconvex region.

\subsection{Optimal Control Problem Formulation and Numerical Solution}

Perhaps the biggest difficulty in formulating an optimal control problem for EST procedures is the presence of the disjunctive path constraint (21). Direct incorporation of this constraint makes the control problem nonsmooth and may cause standard numerical solution procedure to abort prematurely. To remedy this, a multi-stage formulation of the problem is adopted next $[14,30]$. This formulation relies on the observation that the rotor speed $\Omega_{H}(t)$ will decrease monotonically from its rated value $\Omega_{H}^{\mathrm{r}}$ at $t=0$ down to zero at $t_{\mathrm{f}}$ during a typical EST procedure. Therefore, $\Omega_{H}(t)$ will cross the cut-in rotor speed $\Omega^{\text {ci }}$ exactly once. In the optimal control problem $(\mathrm{P})$ below, the extra decision variable $t^{\mathrm{ci}}$ denotes the time 


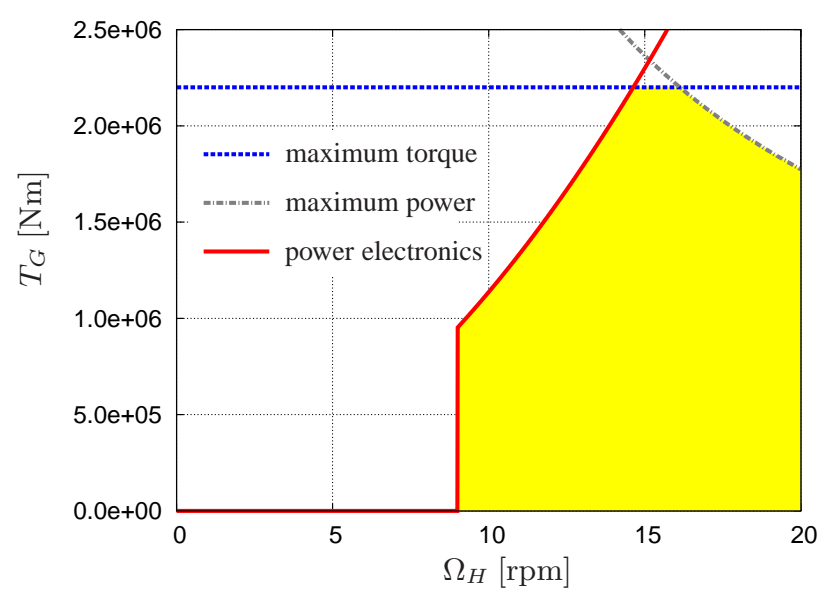

Figure 5. Constraints on torque generator.

when the cut-in speed is reached, that is, $\Omega_{H}\left(t^{\mathrm{ci}}\right)=\Omega^{\mathrm{ci}}$.

$$
\begin{array}{ll}
\min _{\beta_{R}(\cdot), T_{G}(\cdot), t^{\mathrm{ci}}} & \int_{0}^{t_{\mathrm{f}}} \frac{\dot{\Phi}_{T}(t)^{2}}{\sqrt{\dot{\Phi}_{T}(t)^{2}+\epsilon^{2}}} d t \\
\text { s.t. } \quad & \operatorname{model}(7)-(14) \\
& \Omega_{H}\left(t^{\mathrm{ci}}\right)=\Omega^{\mathrm{ci}} \\
& \beta\left(t_{\mathrm{f}}\right) \geq \beta_{F} \\
& 0 \leq T_{G}(t) \leq T_{G}^{\max } \\
& T_{G}(t) \Omega_{H}(t) \leq 1.1 T_{G}^{\mathrm{r}} \Omega_{H}^{\mathrm{r}} \\
& \beta^{\mathrm{br}} \leq \beta(t) \leq 90 \\
& -\dot{\beta}^{\max } \leq \dot{\beta}(t) \leq \dot{\beta}^{\max } \\
& -\ddot{\beta}^{\max } \leq \ddot{\beta}(t) \leq \ddot{\beta}^{\max } \\
& T_{G}(t) \leq a \Omega_{H}(t)\left[\Omega_{H}(t)+b\right], \quad 0 \leq t \leq t_{\mathrm{f}} \\
& T_{G}(t) \leq 0, \quad t^{\mathrm{ci}} \leq t \leq t_{\mathrm{f}} \\
& 0 \leq t^{\mathrm{ci}} \leq t_{\mathrm{f}}
\end{array}
$$

An important parameter to set in $(\mathrm{P})$ is the final time $t_{\mathrm{f}}$. In order to allow for fair comparisons, $t_{\mathrm{f}}$ is here equal to the time it takes the blades to reach the feathered position in a standard EST procedure (constant pitch rate of $\dot{\beta}^{R}=5.5 \mathrm{deg} / \mathrm{s}$ and generator torque is the maximum permissible level dictated by (19)-(21)). Because the rated pitch angle changes with the wind speed $V_{\infty}$, so does $t_{\mathrm{f}}$. A description of the other optimization parameters can be found in Table II.

Table II. Optimization parameters.

\begin{tabular}{crll}
\hline Parameter & \multicolumn{2}{c}{ Value (Case Study) } & Description \\
\hline$\beta_{F}$ & 89.9 & {$[\mathrm{deg}]$} & Feathered position \\
$\Omega^{\mathrm{ci}}$ & 9 & {$[\mathrm{rpm}]$} & Cut-in rotor speed \\
$\beta^{\text {br }}$ & -2 & {$[\mathrm{deg}]$} & Below-rated pitch angle \\
$\dot{\beta}^{\max }$ & 8 & {$[\mathrm{deg} / \mathrm{s}]$} & Pitch rate bound \\
$\ddot{\beta}^{\max }$ & 30 & {$\left[\mathrm{deg} / \mathrm{s}^{2}\right]$} & Pitch acceleration bound \\
$T_{G}^{\max }$ & $2.2 \times 10^{6}$ & {$[\mathrm{Nm}]$} & Maximum torque (at above-rated speed) \\
$a$ & $7.2232 \times 10^{5}$ & {$\left[\mathrm{Nm} \mathrm{s} / \mathrm{rad}^{2}\right]$} & Power electronics constraint parameter \\
$b$ & 0.4558 & {$[\mathrm{rad} / \mathrm{s}]$} & Power electronics constraint parameter \\
$\epsilon$ & $10^{-5}$ & {$[\mathrm{rad} / \mathrm{s}]$} & Tolerance for cost smooth approximation \\
\hline
\end{tabular}


Numerical Solution Procedure A direct numerical procedure is considered subsequently, where the control trajectories are parameterized using a simple function basis, such as piecewise constant or piecewise affine functions. Because the response of the low-order model is nonstiff, the simultaneous approach [31, 15] that uses orthogonal collocation to reduce the optimal control problem to a large-scale nonlinear program (NLP) is well suited, and it is preferred over the sequential approach [13, 14]. Specifically,

- 50 subintervals are used in each stage $\left(0, t^{\mathrm{ci}}\right]$ and $\left(t^{\mathrm{ci}}, t_{\mathrm{f}}\right]$ of the control problem, thus summing up to a total of 100 subintervals;

- A piecewise affine control parameterization, with enforced continuity at each subinterval, is used for both $T_{G}$ and $\beta_{R}$. This is motivated by the fact that a discontinuous control profile contains undesirable high-frequencies that may tap into the neglected higher-order dynamics of the system, thereby invalidating the optimization results;

- Lagrange polynomial approximations are substituted for the state variables in each subinterval, using 4-point Radau collocation;

- The resulting large-scale NLP problem (about 4,800 variables and 4,900 equality/inequality constraints) is solved using the SQP method implemented in the software package SNOPT [32].

The solution time for the NLP with this discretization approach is in the order of a few minutes on a standard laptop computer $^{\S}$.

It should also be noted that since the low-order model (7)-(14) is affine in $T_{G}$ and $\beta_{R}$ and since the cost functional (16) does not depend explicitly on these controls, the optimal control problem $(\mathrm{P})$ is singular with respect to either controls. Such singularity typically results in the presence of discontinuities in the optimal control, including bang-bang control arcs [33]. Moreover, the sensitivity of the optimal cost to the singular controls is usually low, requiring many iterations for the NLP solver to converge when fine control parameterization is used.

The optimal results obtained with this approach are discussed in the following sections. A case study is detailed in Sections 4 and 5, which considers favorable shut-down scenarios where the wind turbine is operating properly and the actuators and sensors are not faulty. Extensions to more challenging shut-down scenarios are discussed later in Section 6.

\section{OPTIMAL PROCEDURES FOR RAPID SHUT-DOWN}

\subsection{Solutions of the Original Singular Control Problem}

Optimal solutions of the singular control problem (P), as computed for various above-rate wind speeds $V_{\infty}=18,22$ and $26 \mathrm{~m} / \mathrm{s}$, are shown in Figure 6. While it is clear that the optimal trajectories are quite sensitive to $V_{\infty}$, it is also noted that the general structure or pattern of these trajectories is conserved across different $V_{\infty}$ values.

The rotor velocity $\Omega_{H}$ decreases from its rated value $\Omega_{H}^{\mathrm{r}}$ to a value close to zero, and so does the generator torque $T_{G}$. Interestingly, $T_{G}$ stays at its maximum allowable value along the entire optimal path, starting at $T_{G}^{\max }$, then decreasing along the path constraint by taking values $a \Omega_{H}(t)\left[\Omega_{H}(t)+b\right]$, and finally switching to zero at $t^{\mathrm{ci}}$ as soon as the cut-in rotor speed is reached.

The major effect of different wind speed values $V_{\infty}$ appears to be shifting the optimal collective pitch reference trajectories $\beta_{R}(t)$. In all cases, the pitch trajectories reach the specified feathered position after a rather regular increase from their respective rated pitched angles-see the discussion on initial conditions in Subsection 2.1. In contrast, the optimal pitch rate response $\dot{\beta}$ exhibits spurious variations in spite of the choice of a continuous, piecewise-affine parameterization for the pitch reference $\beta_{R}$, and so does $\ddot{\beta}$ (not shown). The presence of these high-frequencies in the collective pitch rate and acceleration, which is caused by the singularity of the control problem, is susceptible to invalidation of the low-order model and shall be the focus of further refinement in Subsection 4.2.

Regarding load reduction, it is evident from the middle and right lower plots in Figure 6 that the optimal control strategies are particularly efficient at dampening the fore-aft tower displacement. The first compressive peak brings the tower back to nearly vertical position, where it stays during the remainder of the EST horizon. This observation is confirmed in Figure 7, which shows a comparison of the optimal and standard EST procedures at $V_{\infty}=22 \mathrm{~m} / \mathrm{s}$. The oscillations in the pitch reference $\beta_{R}$ appear to be playing a key role in maintaining the fore-aft displacement close to vertical position from $t=1 \mathrm{~s}$ onwards. Quantitatively, the first compressive peak is significantly reduced, with (negative) maximal displacements of $-5.1 \mathrm{~cm},-4.0 \mathrm{~cm}$ and $-2.6 \mathrm{~cm}$ with the optimal EST procedure at $V_{\infty}=18,22$ and $26 \mathrm{~m} / \mathrm{s}$, respectively, compared to maximal displacements of $-12.9 \mathrm{~cm},-12.0 \mathrm{~cm}$ and $-10.0 \mathrm{~cm}$ with the standard EST procedure.

Observe also from the lower right plot in Figure 6 that the tower shows small fore-aft oscillations near the end of the time horizon. In practice, these residual oscillations can last over several minutes-see Subsection 4.3 for an illustration.

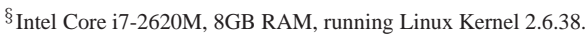



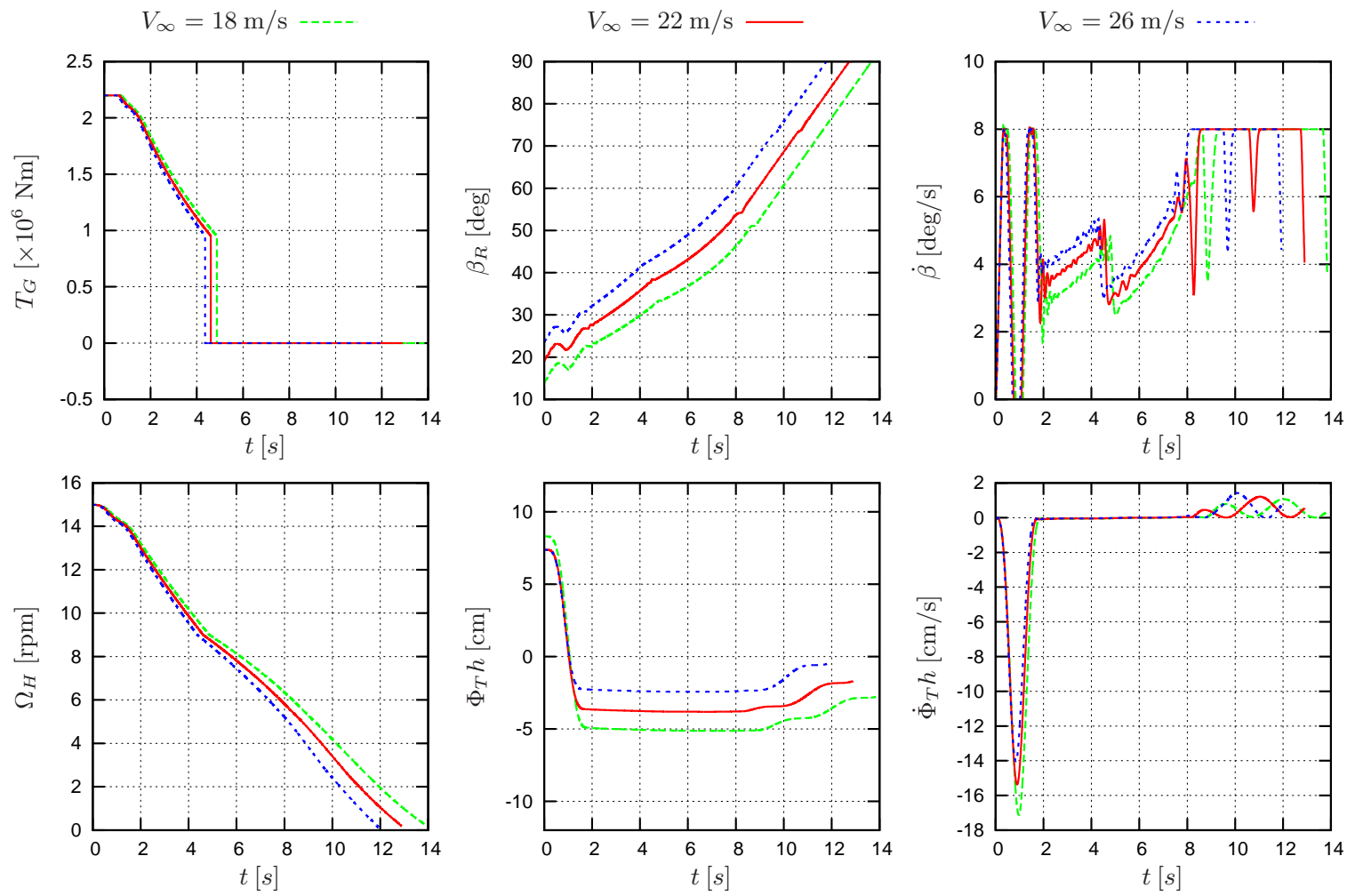

Figure 6. Optimal controls and responses of (P) at $V_{\infty}=18,22$ and $26 \mathrm{~m} / \mathrm{s}$.

This is because once the pitch has reached its feathered position and the rotor speed is below its cut-in value, neither aerodynamic nor mechanical damping are possible anymore; that is, the WTG can no longer be controlled.
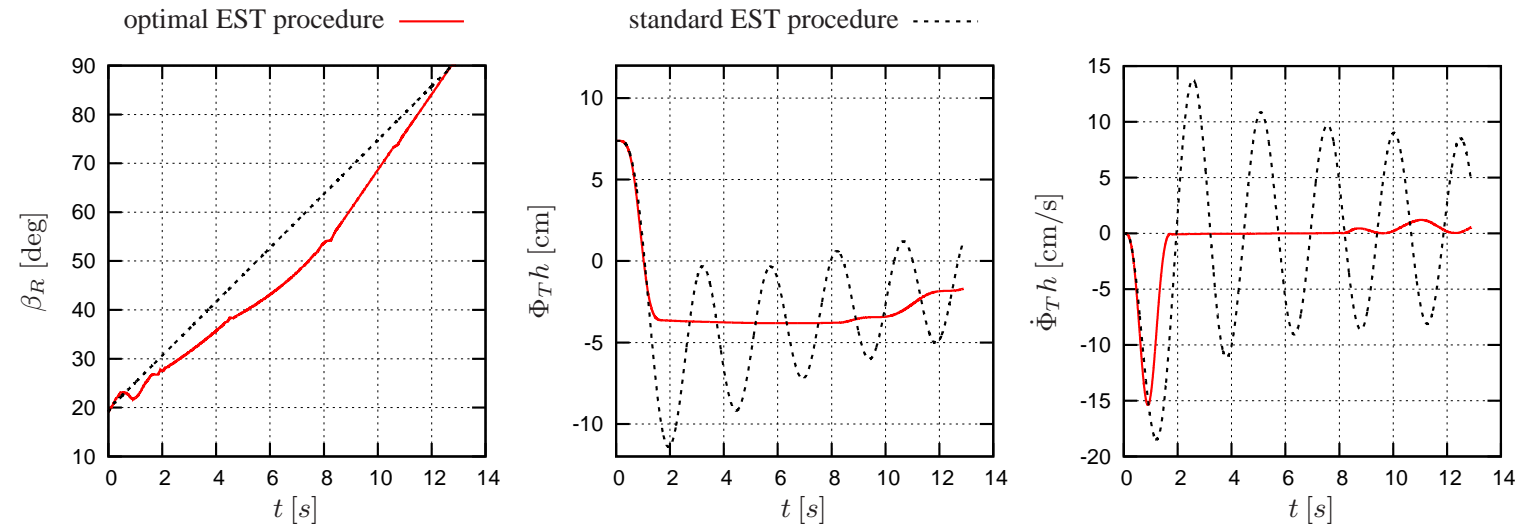

Figure 7. Comparison of the optimal and standard (constant pitch rate) EST procedures at $V_{\infty}=22 \mathrm{~m} / \mathrm{s}$.

\subsection{Regularization and Simplification of the Optimal Control Problem}

Despite their large potential for extreme load reduction, the optimal EST strategies in Figure 7 are inevitably degraded when implemented in the Bladed simulation environment - the results of this validation shall be presented later in Subsection 4.3. This behavior can be attributed to the presence of high-frequencies in the collective pitch rate and acceleration profiles, which invalidate the low-order model, together with the use of the approximate metric (15) for minimizing structural load and other modeling assumptions. 
This situation can be remedied in either one of two ways. Extending the low-order model (7)-(14) by accounting for neglected modes is one possibility, but it makes the optimal control problem computational more challenging to solve. Moreover, many extra modes may be necessary in order to make the predictions reliable enough. Another possibility is to regularize the optimal control problem by adding a secondary objective to the problem that penalizes high-frequencies in the collective pitch dynamics. This latter approach is adopted here, with the following regularization objective

$$
R=\int_{0}^{t_{\mathrm{f}}} \ddot{\beta}(t)^{2} d t=\int_{0}^{t_{\mathrm{f}}}\left[K_{P}\left(\beta_{R}(t)-\beta(t)\right)-K_{D} \dot{\beta}(t)\right] d t .
$$

This extra objective term is also interesting in that it removes the singularity of the problem in the control variable $\beta_{R}$. It should be noted, at this point, that such a choice for the regularization term is by no means unique. For instance, one could consider a more general term in the form of a high-pass filter as well, although this then requires extra tuning parameters.

A simple technique to deal with the resulting multi-objective problem consists in aggregating both objectives as a single objective to be used in lieu of (16) in (P),

$$
J=\int_{0}^{t_{\mathrm{f}}} \frac{\dot{\Phi}_{T}(t)^{2}}{\sqrt{\dot{\Phi}_{T}(t)^{2}+\epsilon^{2}}} d t+\omega \int_{0}^{t_{\mathrm{f}}} \ddot{\beta}(t)^{2} d t,
$$

with $\omega \geq 0$ the regularization parameter. The Pareto front for non-dominated solution in the multi-objective optimal control problem can be generated by varying $\omega$ in a wide range. One such front in the case of $V_{\infty}=22 \mathrm{~m} / \mathrm{s}$ is represented by the dotted blue line on the left plot in Figure 8.

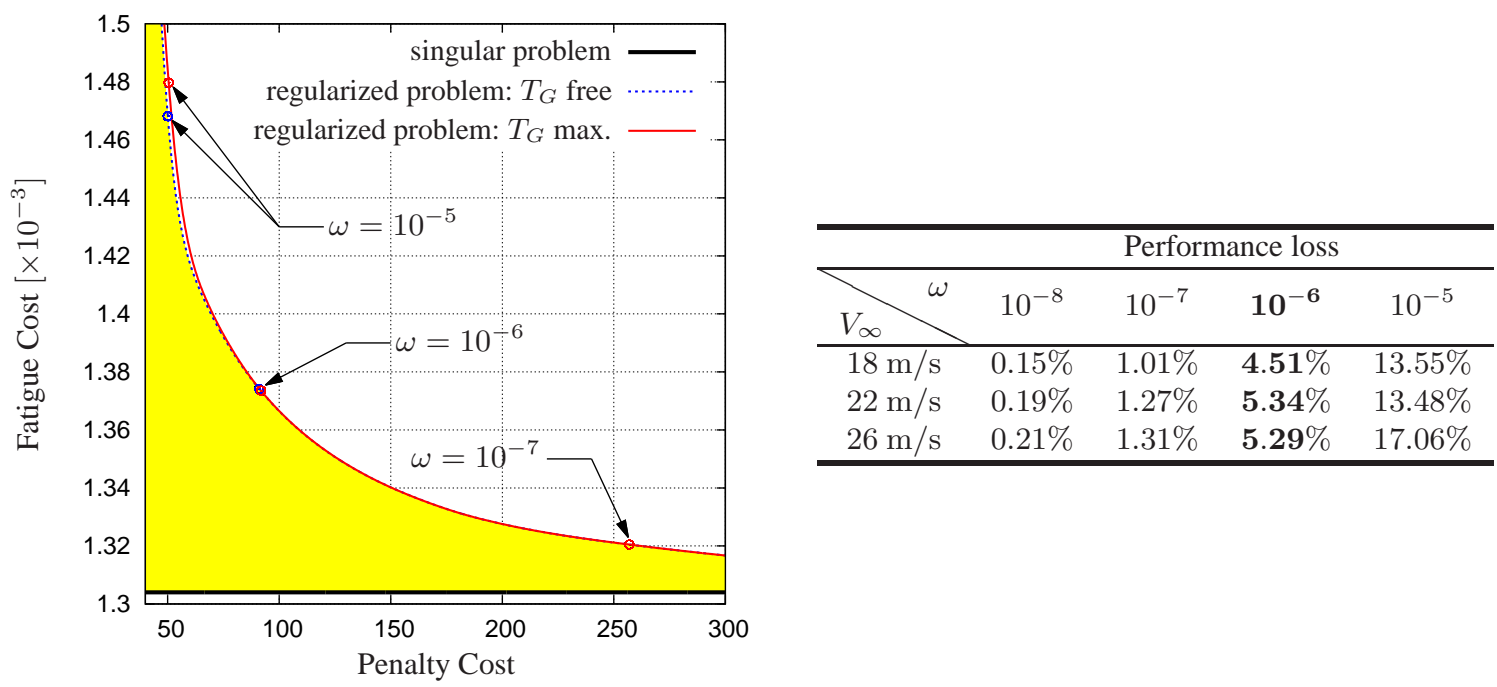

Figure 8. Left plot: Pareto curve of fatigue reduction vs. regularization at $V_{\infty}=22 \mathrm{~m} / \mathrm{s}$. Right table: Performance loss, expressed in terms of the cost function (16), for various regularizations and free-flow wind speeds.

Another interesting insight gained from the results in Subsection 4.1 is that $T_{G}$ stays at its maximum feasible value in the optimal solution, regardless of the free-flow wind speed $V_{\infty}$. Therefore, the decision was made to simplify the optimal control problem $(\mathrm{P})$ by fixing $T_{G}$ at its maximum value as

$$
T_{G}(t)= \begin{cases}\min \left\{T_{G}^{\max }, a \Omega_{H}(t)\left[\Omega_{H}(t)+b\right]\right\}, & \text { if } \Omega_{H}(t) \geq \Omega^{\mathrm{ci}} \\ 0, & \text { otherwise },\end{cases}
$$

thereby leaving $\beta_{R}$ as the only free control variable. The results of this simplification can be seen on the left plot in Figure 8, with the corresponding Pareto front in solid red line. Note that the solutions of the problem with and without fixing $T_{G}$ at its maximum value are extremely close, so this latter simplification causes a minimal loss in performance.

In contrast, the performance loss caused by the regularization term in the modified cost function (23) grows quite rapidly as the regularization parameter $\omega$ increases. A few values are reported on the left plot in Figure 8, and a systematic comparison is shown in the table opposite for $V_{\infty}=18,22$ and $26 \mathrm{~m} / \mathrm{s}$. The choice of $\omega=10^{-6}$ limits the performance loss to around $5 \%$ with respect to the original singular solution of $(\mathrm{P})$, and it can be verified from the left plot in Figure 9 that this regularization significantly dampens the pitch rate variations compared to the singular case. Observe also from the middle plot in Figure 9 that the magnitude of the first compressive peak remain seldom affected by selecting $\omega=10^{-6}$.

The final optimal trajectories for the regularized and simplified control problem are displayed in Figure 10. A validation of these EST procedures in the Bladed simulation environment is conducted in the next subsection. 
Singular $-\omega=10^{-7} \ldots \ldots$
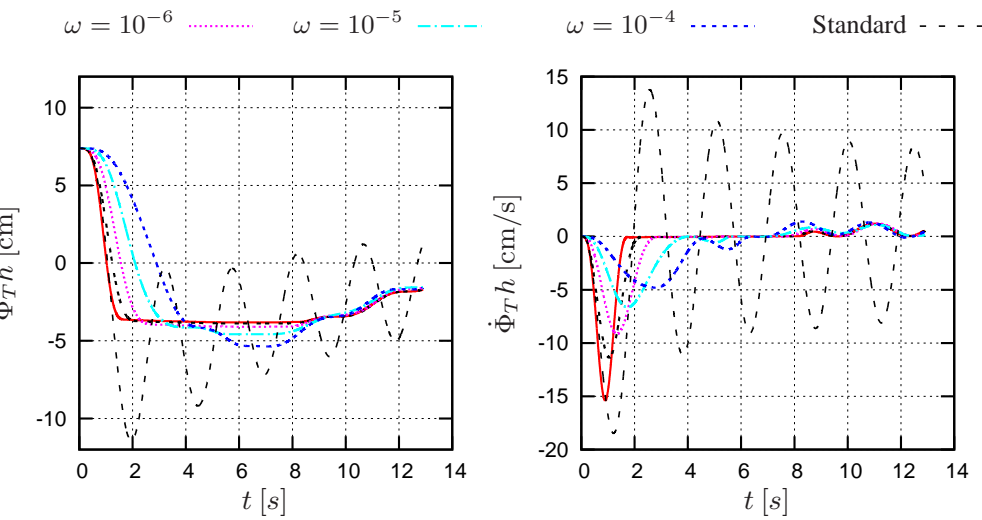

Figure 9. Comparison of optimal EST procedures at $V_{\infty}=22 \mathrm{~m} / \mathrm{s}$ for various regularizations.
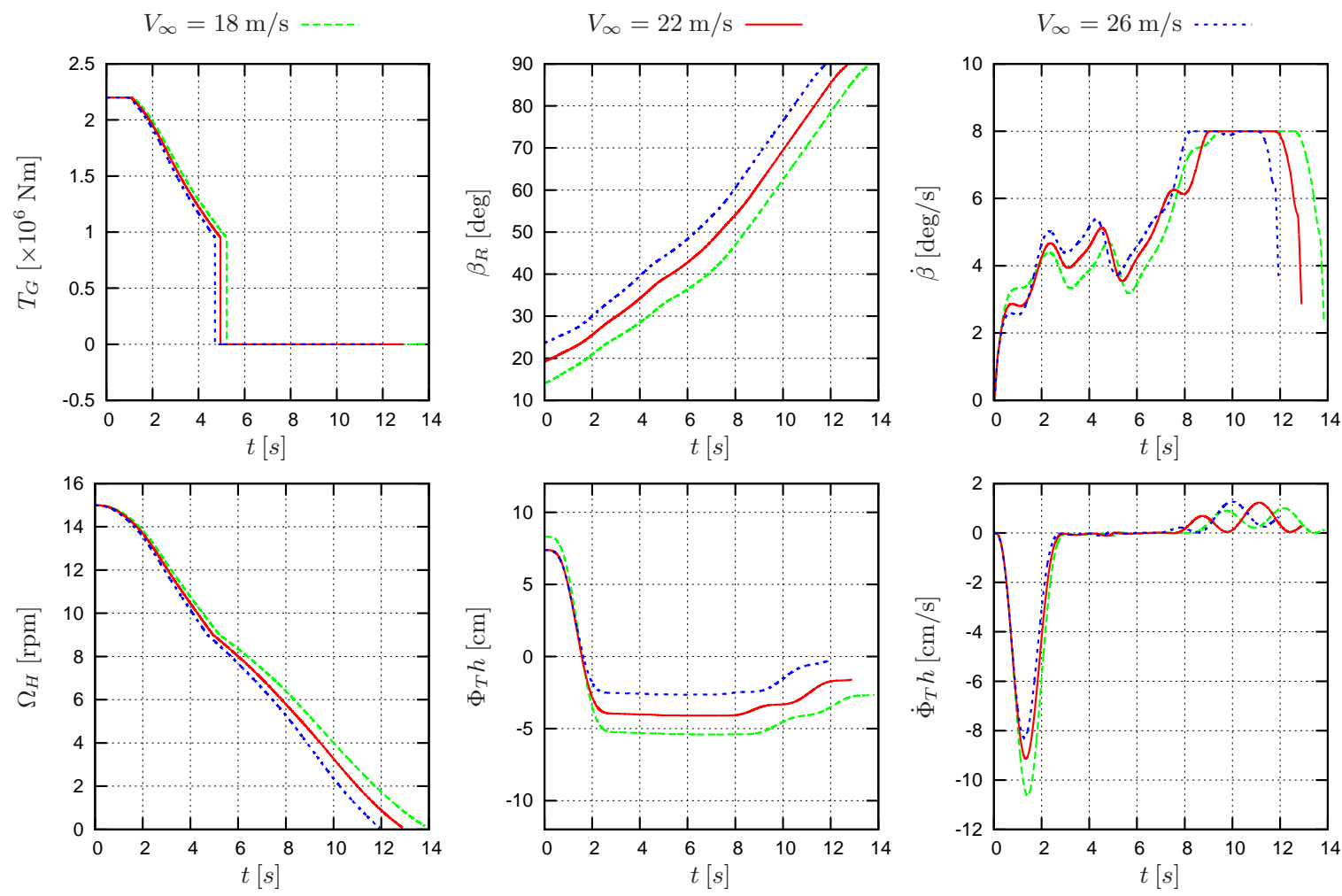

Figure 10. Optimal controls and responses of (P) with regularization $\omega=10^{-6}$ at $V_{\infty}=18,22$ and $26 \mathrm{~m} / \mathrm{s}$.

\subsection{Validation of the Optimized EST Procedure in the Bladed Environment}

The Bladed simulation environment is used in this subsection to validate the optimized EST strategies and compare them with the standard (constant pitch rate) procedures. The comparisons below consider laminar wind conditions, unless otherwise noted.

Presented in Figure 11 is a comparison, where the optimized EST procedures have been computed for an above-rated wind speed of $V_{\infty}=22 \mathrm{~m} / \mathrm{s}$ and are applied at the same nominal wind speed of $22 \mathrm{~m} / \mathrm{s}$. Large reductions in the foreaft displacement oscillations (top left plot) and in the corresponding tower root moment (top right plot) are obtained for different regularizations, although these oscillations turn out to be bigger than those initially predicted by the low-order model (see Figure 9); such discrepancies result from the neglected out-of-plan blade modes and higher fore-aft tower modes, as already noted in Subsection 2.2. The bottom plot in Figure 11 shows the same simulation results as the top right 
plot, but on a longer time horizon of $140 \mathrm{~s}$. Because both the mechanical and aerodynamic dampening of the tower become very small as soon as the collective pitch gets close to the feathered position and the rotor speed gets close to zero, the residual oscillations of the tower typically persist for several minutes.

In the top right plot, the first compressive peak and subsequent compressive/tensile peaks are seen to be reduced significantly, with tower root moments between $-5 \times 10^{6}$ and $2 \times 10^{6} \mathrm{Nm}$, compared to moments between $-20 \times 10^{6}$ and $11 \times 10^{6} \mathrm{Nm}$ with standard pitch control. Similar load reductions are obtained for case studies at above-rated wind speeds of 18 and $26 \mathrm{~m} / \mathrm{s}$ through optimization of the collective pitch reference.
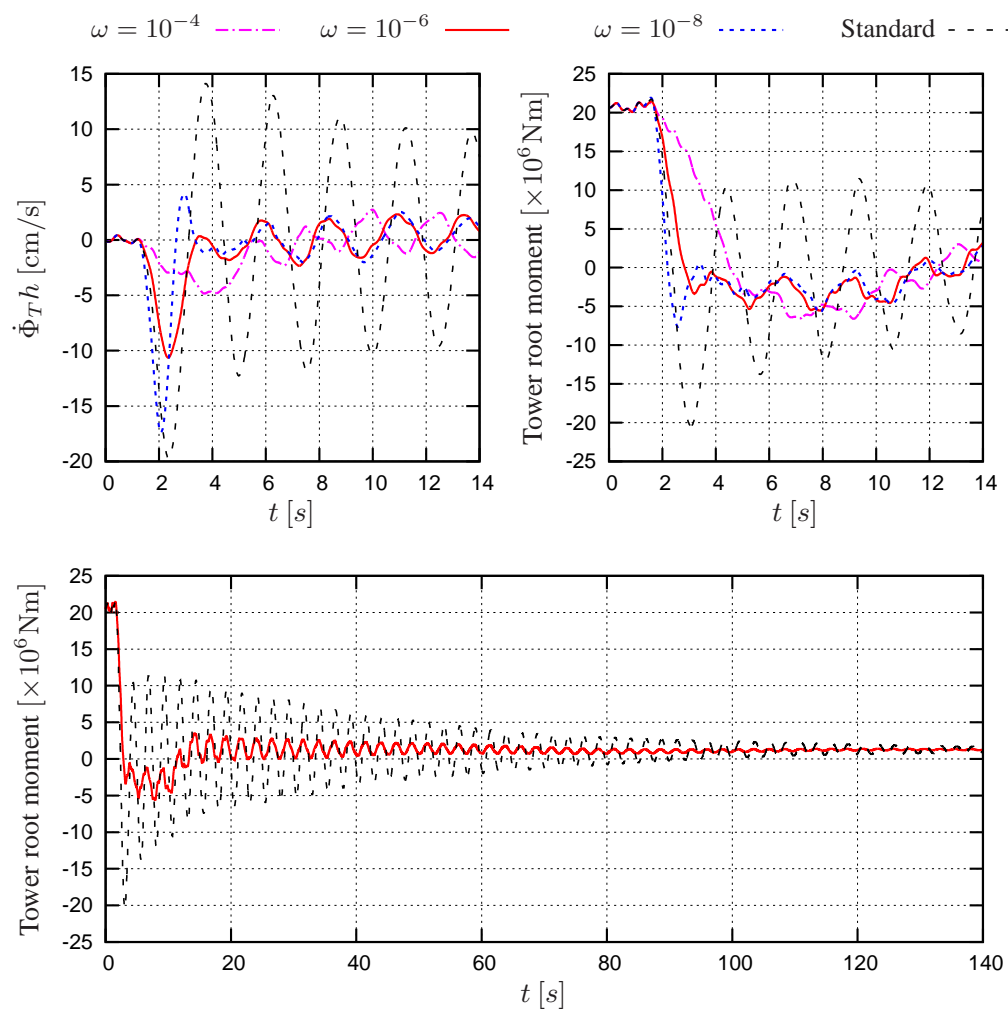

Figure 11. Comparison between a standard (constant pitch rate) EST strategy and optimized EST strategies for $V_{\infty}=22 \mathrm{~m} / \mathrm{s}$, at a laminar wind speed of $22 \mathrm{~m} / \mathrm{s}$, in the Bladed simulation environment.

The performance of an EST strategy, optimized for a nominal wind speed of $V_{\infty}=22 \mathrm{~m} / \mathrm{s}$ (with a regularization of $\omega=10^{-6}$ ), but now applied under perturbed (laminar) wind conditions of 20 and $24 \mathrm{~m} / \mathrm{s}$, is shown on the left and middle plots in Figure 12, respectively. Such perturbations are found to reduce the benefit of the optimization significantly, compared to the reductions obtained under nominal wind conditions (Figure 11), and can even lead to larger structural loads than with standard pitch control. The reason for this is twofold. Not only does a change in the wind-speed have a direct influence on the dynamics, but it also influences the (steady-state) initial conditions, and it is found that this latter contribution typically has a larger influence than the former. The right plot in Figure 12 displays the performance of the same EST strategy, now applied for an average wind speed of $V_{\infty}=22 \mathrm{~m} / \mathrm{s}$ under turbulent conditions ${ }^{\mathbb{T}}$ and with a wind gust occurring as the EST is triggered (worst case). In this scenario too, a significant increase of the tower root moment is observed.

Notwithstanding the potential of optimization-based pitch control for extreme load reduction, these results clearly underpin the need for implementing an on-line control scheme, where the collective pitch control reference can be adapted in order to compensate for variations in the wind conditions. On such scheme is developed and tested in the following section.

T The following settings are used in the Bladed simulation environment: IEC-2, NTM 

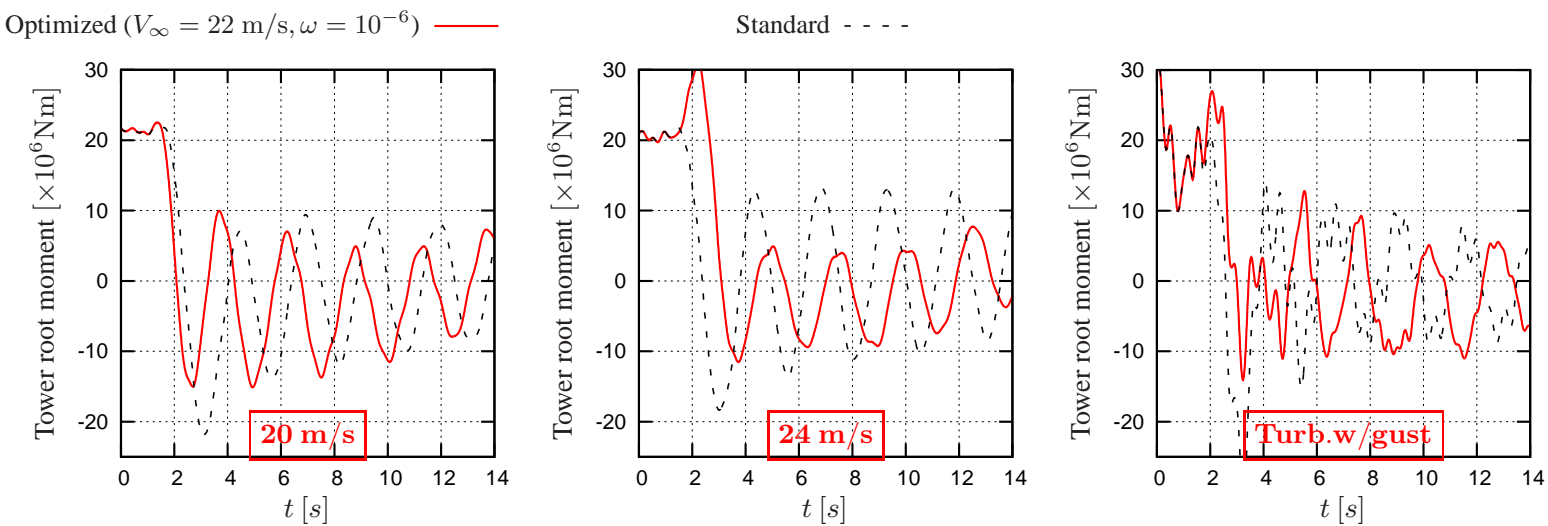

Figure 12. Comparison between standard (constant pitch rate) and optimized EST strategies for $V_{\infty}=22 \mathrm{~m} / \mathrm{s}$, under perturbed wind conditions, in the Bladed simulation environment.

\section{REAL-TIME OPTIMAL CONTROL OF RAPID SHUT-DOWN PROCEDURES}

Fast Sensitivity-Update Procedure Mid-course control corrections are particularly important in the presence of wind gusts or other fast changing conditions. Nonetheless, recomputing accurate solutions to the optimal control problem (P) repeatedly is a computationally demanding task, which is hardly compatible with the fast dynamics involved in WTG control. Of the alternatives, a possible approach involves precomputing and storing a number of optimal control profiles for various free-wind scenarios, and then selecting the ones that are closest to the actual wind speed when the EST is triggered. Perhaps the biggest limitation with this simple interpolation approach is that a large number of scenarios might be needed to guarantee a good performance in all events, and this quickly becomes unmanageable when mid-course corrections are to be performed during the EST as well.

A more practical approach involves precomputing optimal solutions for a reduced number of free-wind-speed scenarios, and then using the available on-line measurements to track the nearest optimal response by adapting the pitch reference during run time. For instance, the optimal tower acceleration profile can serve as a reference in a linear parameter-varying (LPV) feedback controller, based on the nacelle fore-aft acceleration on-line measurements [7]. However, the design of such robust linear controller might prove challenging given the rapid changes in process dynamics during an EST, such as the rapid decrease in tip speed ratio $\lambda$ with decreasing rotor velocity $\Omega_{H}$. Moreover, it is well known that the tracking of an optimal response under perturbed conditions can possibly lead to large performance loss, here in terms of structural load reduction.

Fast sensitivity update, also known as neighboring extremal control, is a technique whereby an auxiliary optimization problem is formulated via linearizing the original OCP around a given optimal solution [20, 21, 34, 22, 35], as obtained under a particular free-wind speed and initial state scenario in the present case. This approach mitigates the performance degradation inherent to linear feedback by accounting for the optimization objective in the controller formulation, and it is applied to the EST problem subsequently. In the case that the OCP has been discretized using control parameterization, the auxiliary optimization problem can be cast as a quadratic program (QP), which is typically solved in less than 1-10 ms on a standard CPU [36]. It is the repeated solution of this QP that provides the required (discrete) feedback law at high sampling frequencies $(100 \mathrm{~Hz}-1 \mathrm{kHz})$. Note that it is also possible to account for the system nonlinearities in the control corrections by re-compute the OCP linearization on-line based on the latest estimates of the model states and parameters. In particular, with recent advances in real-time iteration schemes that rely on automatic code generation, such on-line corrections have been demonstrated at high frequencies (up to $1 \mathrm{kHz}$ ) for small- to medium-scale mechanical systems [37, 38].

The constraints in the sensitivity QP are obtained by linearizing the constraints in the NLP (discretized OCP (P)), whereas the QP objective involves the second-order derivatives of its Lagrangian function. The required first- and secondorder derivatives can be precomputed off-line in a number of free-wind speed and initial state scenarios. At this point, it should be noted that the objective and constraint functions in the sensitivity QP also depend linearly on a number of perturbation parameters, which correspond to the deviations in free-wind speed and initial state. Provided that these perturbations can either be measured or estimated at the beginning of an EST procedure (see below), the solution for the sensitivity QP then provides the corrective control actions in each time subinterval.

- In most recent WTGs, the state variables $\ddot{\Phi}_{T}, \Omega_{H}, \beta$, and $\dot{\beta}$ are readily available or can be deduced from sensor measurements. The tower deformation $\Phi_{T}$ too can be estimated if tower strain gauge sensors are available, or 
estimated based on observation techniques such as extended Kalman filtering (EKF) or moving-horizon estimation (MHE) [39, 40].

- Reliable measurements of the free-wind velocity $V_{\infty}$ are often unavailable. Therefore, $V_{\infty}$ has to be estimated from observation techniques; see, e.g., [41].

In the subsequent case study, it is assumed that (estimates of) these initial states and parameters are available. The combination of the proposed fast-sensitivity update approach with state/parameter estimators is beyond the scope of this paper and shall be investigated as part of future work.

Case Study Results of the fast sensitivity-update procedure described previously are now presented and comparisons are made in the Bladed simulation environment for validation. For simplicity, the control is only corrected at the start of the EST; that is, no mid-course corrective control actions are taken here. To compute the correction, an estimate of the free-wind speed variation $\Delta V_{\infty}$ is obtained at initial EST time by linearizing the initial condition (5) based on the rated values $\Omega_{H}^{\mathrm{r}}, T_{G}^{\mathrm{r}}$ and $\beta^{\mathrm{r}}$ and the corresponding measurements for $\Omega_{H}(0), T_{G}(0)$ and $\beta(0)$. Also note that since tower strain gauge sensors are considered to be unavailable in the simulation environment Bladed, the tower position deviation $\Delta \Phi_{T}(0)$ cannot be estimated accurately and is taken equal to zero here.

A comparison between adapted and standard EST strategies is presented in Figure 13. The adaptation is based on a sensitivity QP designed for a nominal free-wind speed of $V_{\infty}=22 \mathrm{~m} / \mathrm{s}$ (with a regularization of $\omega=10^{-6}$ ), and the perturbed conditions correspond to laminar wind conditions of $20 \mathrm{~m} / \mathrm{s}$ (left plot) and $24 \mathrm{~m} / \mathrm{s}$ (right plot). The large reductions in terms of extreme loads, as compared to the left and middle plots in Figure 12, confirm the efficiency of the proposed QP corrective approach. These results also indirectly confirm that the low-order model is well suited for on-line optimization purposes.
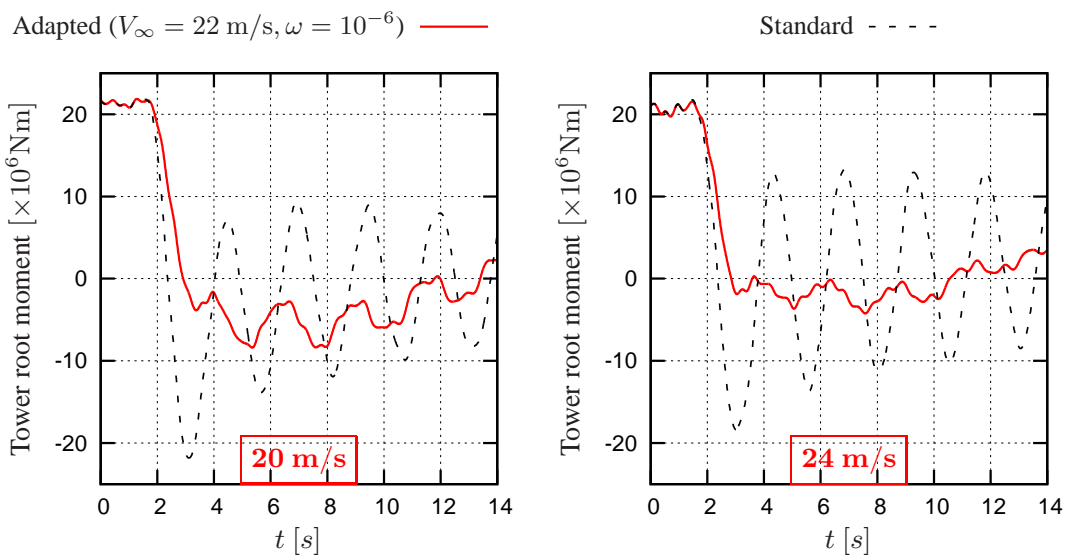

Figure 13. Comparison between standard (constant pitch rate), reoptimized, and adapted (sensitivity QP) EST strategies for $V_{\infty}=22 \mathrm{~m} / \mathrm{s}$, under perturbed wind conditions, in the Bladed simulation environment.

\section{DISCUSSIONS}

The developments in Sections 4 and 5 assume that, in carrying out the EST procedure, the actuators and sensors of the WTG are not faulty and that the main components of the WTG, including the generator and the blades, are operating properly. While EST procedures can be triggered under such favorable conditions, they might as well be triggered after a WTG component has become unavailable or happen when sensors are faulty or unavailable. A number of common scenarios inspired from the standard IEC 61400-1 [10] can be accommodated in the proposed optimization and control framework and are discussed subsequently. Quite clearly, there also are more severe scenarios which cannot be tackled solely through optimization and control. This includes (i) loss of computational power or of the control cabinet, (ii) total loss of pitch control, or (iii) serious damage of the wind turbine structural integrity. Alternative shutdown procedures by means other than pitch or torque control should be considered under these scenarios, such as the use of one or several fail-safe tip brakes [42]. However, this falls beyond the scope of the paper.

In conducting an EST when some components or sensors are undergoing failure, it is paramount that all of the problems are properly identified. Condition monitoring systems that assess the WTG operational status are well developed, as well 
as fault detection and isolation systems that detect excessive vibration or gearbox failure; see, e.g., [43] for a review. In addition, nonlinear state estimation techniques such as MHE provide an ideal framework to tackle the detection and isolate of actuators and sensors faults [39,40], and could become the norm for WTGs in future years. These methods also have the ability to detect parameter drifts, which could be useful for monitoring blade icing or component failures, and can detect/handle sensor failure in the case that redundant measurement information is available, for instance by removing or minimizing the weight of the faulty sensor [40]. However, further investigation is clearly warranted.

Loss of Generator Torque A common scenario triggering EST procedures is loss of generator torque. This situation can arise in the case of (i) grid loss, (ii) power electronics failure, or (iii) generator/gearbox mechanical failure. Loss of generator torque typically produces an overspeed alarm, which then triggers the EST procedure. If the generator torque is not available to perform the EST procedure, essentially the same optimization formulation and solution approach as described in Sections 4 and 5 can be used, but with the torque constraints (19)-(21) in Problem (P) now replaced by

$$
T_{G}(t)=0, \quad 0 \leq t \leq t_{\mathrm{f}}
$$

Moreover, the optimization problem further simplifies by eliminating the switching time $t^{\mathrm{ci}}$ for the decision variables.

The results of an EST scenario with loss of generator torque are shown in Figure 14, everything else being the same as in the case study of Subsection 4.2. It is found that even when no torque can be reclaimed from the generator, a pitch-rate control profile can be found that dampens the fore-aft tower displacement efficiently, thereby alleviating extreme structural loads. Although rather expectedly, a comparison with the results in Figures 10 reveals that the negative load peak would be significantly larger in this scenario, as the rotor speed keeps increasing during the first 1-2 seconds after the shut-down is triggered. The residual oscillations near the end of the horizon are also larger.
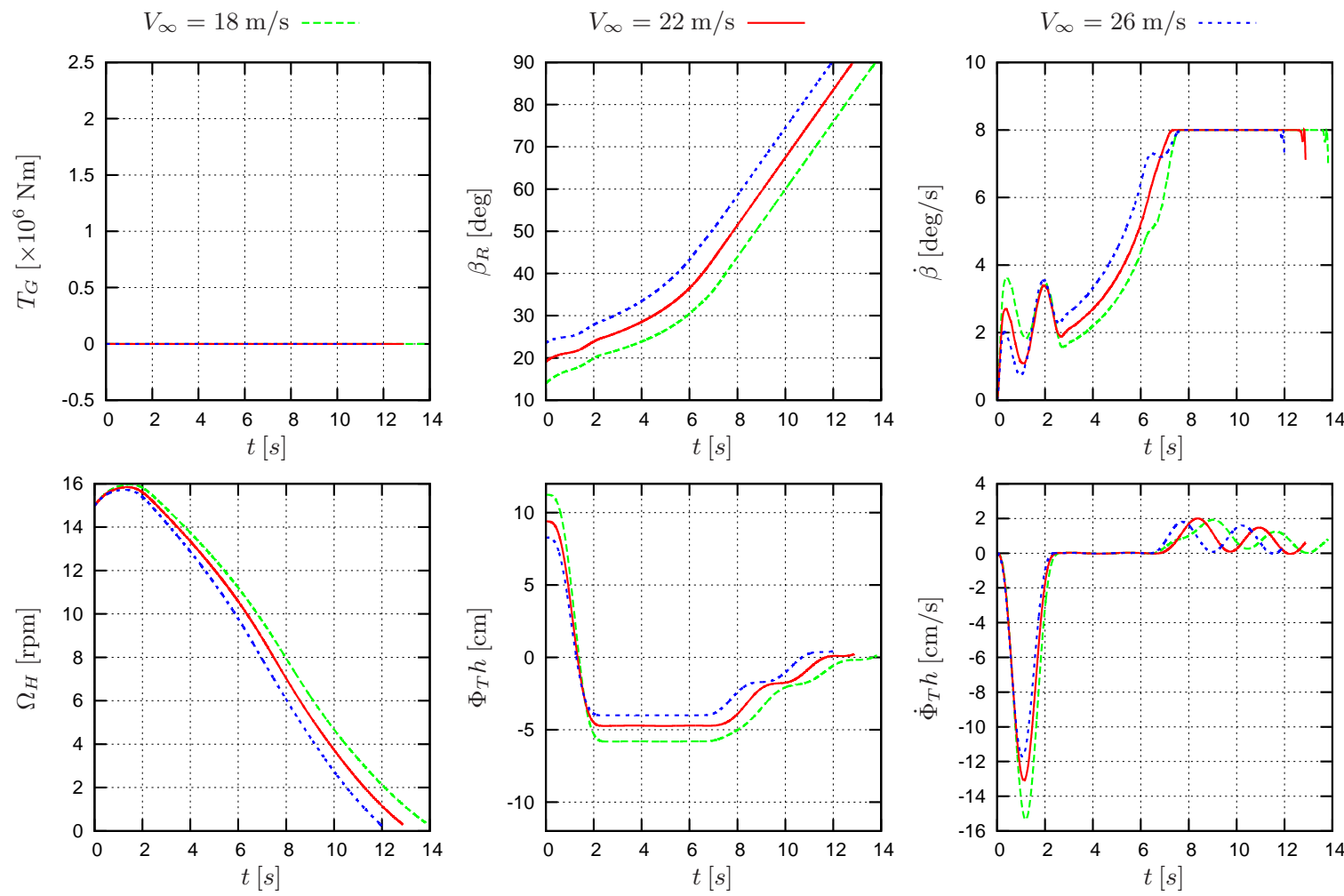

Figure 14. Optimal EST procedures with loss of generator torque. All of the other parameters are the same as for the case study in Figure 10.

Loss of Pitch Control The loss of pitch control of an individual blade can trigger an EST procedure. A typical scenario is a blade being stuck in a fixed position, while the two other blades remain operational. While the proposed methodology is developed for collective pitch control, it can be extended to encompass individual pitch control. The idea would be to 
treat the individual pitch rates as three independent sets of decision variables in Problem $(\mathrm{P})$ and then set the pitch rate of the blade undergoing failure to zero. Wind turbine models involving individual pitch control are available in the literature [2] and could be used to develop a reduced model similar to the model (7)-(14). Both the simulators Bladed [26] and FAST [44], for instance, allow simulation of such scenarios.

Blade Icing ESTs can be triggered by hazardous events such as blade icing since the extra mass of the blades can endanger their structural integrity. When ice accumulates on the blades, the mechanical response of a WTG changes and its aerodynamic behavior too may be affected; consequently, the validity of the wind turbine model will decrease. In principle, an estimate of the extra mass of the blades could be inferred based on indirect measurements, for instance by using MHE. On the other hand, inferring and accounting for changes in aerodynamic behavior might prove to be a much more challenging task. The potential of the proposed approach to deal with EST triggered by blade icing is thus unclear and would deserve further investigations.

\section{CONCLUSIONS AND FUTURE DEVELOPMENTS}

The main objective of this paper was to develop a model-based optimization methodology for the reduction of extreme loads during EST events, with emphasis on both off-line and on-line optimization aspects. The problem has been formulated as an optimal control problem, based on a tailored, low-order dynamic model that accounts for the first fore-aft tower mode of a WTG. It has been established that, under favorable shut-down scenarios where the WTG is operating properly and the actuators and sensors are not faulty, tower loads could be significantly alleviated. The case study of 3MW WTG in the Bladed simulation environment has confirmed that large reductions of the first compressive peak and subsequent compressive/tensile peaks of the tower load pattern are obtained at various above-rated wind speeds compared to normal pitch control shutdown, thereby providing a proof-of-concept for such model-based optimization approach.

Next, a fast sensitivity update has been investigated to handle such uncertainties as wind speed variations and estimation error as well as model mismatch. Rather than tracking the optimal response trajectories for one of the precomputed scenarios, this approach solves a sensitivity-based QP repeatedly and leads to a self-optimizing feedback law. Such QPs are parametric in both the wind speed and the initial state and can thus be designed off-line based on the knowledge of the corresponding (nominal) optimal control and response. This way, the on-line burden is restricted to solving the QP, a task that requires limited computational power for small- to medium-scale QPs and can conveniently be repeated at high frequencies in the order of $100 \mathrm{~Hz}-1 \mathrm{kHz}$.

As part of future work, first and foremost is the need to investigate more severe EST scenarios, including cases where part of the sensors and actuators are faulty or unavailable as well as cases of generator torque or pitch control loss, possibly in combination; these issues have been touched upon in the foregoing discussion section. Further improvements and investigations should also be pursued with regards to the optimization methodology itself, including the derivation/use of a higher-order dynamic model, the ability to account for ultimate loads and for a more flexible shut-down horizon, and the application of alternative adaptation/robustification strategies.

The second major structural mode - that is, the first out-of-plane mode corresponding to the in-phase fore-aft oscillation of the three blades - could be taken into account in the optimization problem in either one of two ways: (i) by directly incorporating these extra dynamics in the low-order model so that the resulting optimal controls reduce their influence on the structural fatigue; or (ii) by treating these dynamics in the form of uncertain parameters and then solving the robust optimization problem counterpart to reduce their effect on the optimal solution. Besides, the blades structural fatigue could also be included in the objective function in a form similar to the one used for tower fatigue. Naturally, an arbitrary number of higher-order modes could be accounted for in the optimization problem in a similar way.

Throughout the paper, the EST time horizon $t_{\mathrm{f}}$ has been treated as a parameter, which is determined by the free-wind speed $V_{\infty}$ for convenience. There is no doubt, however, that further fatigue reductions could be achieved by treating $t_{\mathrm{f}}$ as an extra degree of freedom in the optimization problem. Because slowing down the EST procedure tends to reduce the residual structural oscillations by keeping the system controllable over a longer period of time, the optimal $t_{\mathrm{f}}$ may end-up being quite large or even unbounded. In particular, special attention should then be paid to modifying the objective function in such a way that it becomes independent of $t_{\mathrm{f}}$, for instance by using some form of scaling.

Finally, alternative adaptation strategies to the fast sensitivity update in Subsection 5 could be investigated, including reoptimization and parametric programming approaches. It is unclear at this stage whether a more rigorous optimization approach — that is, one that does not need linearize the optimal control problem-would lead to significant improvements, especially if one considers that the results of the fast sensitivity update approach are already quite close to those given by the reoptimization. Another point of interest would be to formulate a robust/stochastic optimization problem counterpart by treating $V_{\infty}$ as a bounded/random variable, thereby reducing the sensitivity of the optimal solution to wind-speed variations and estimation error. 


\section{ACKNOWLEDGEMENT}

The authors are grateful to the reviewers for the thoughtful comments that lead to substantial improvement of the article. Most of the work in the paper was done when SG was with MLS Intelligent Control Dynamics, Glasgow (UK). The authors are especially grateful to MLS for lending them a Bladed license. Special thanks also go to Ms Jessica Monteith for her careful proofreading of the paper and useful suggestions.

\section{REFERENCES}

1. Caselitz P, Kleinkauf W, Kruger T, Petschenka J, Reichard M, Storzel K. Reduction of fatigue loads on wind energy converters by advanced control methods. European Wind Energy Conference, 1997.

2. Bossanyi EA. Further load reduction with individual pitch control. Wind Energ. 2005; 8(4):481-485.

3. Bossanyi EA. Wind turbine control for load reduction. Wind Energ. 2003; 6(3):229-244.

4. Leithead WE, Connor B. Control of variable speed wind turbines: Design task. Int. J. Control 2000; 73(13):11891212.

5. Bossanyi EA. Individual blade pitch control for load reduction. Wind Energ. 2003; 6(2):119-128.

6. Selvam K, Kanev S, Van Wingerden JW, Van Engelen T, Verhaegen M. Feedback-feedforward individual pitch control for wind turbine load reduction. Int. J. Robust Nonlin. Control 2009; 19:72-91.

7. Bossanyi EA. The design of closed loop controllers for wind turbines. Wind Energ. 2000; 3(3):149-163.

8. Gardner P, Garrad A, Falbe-Hansen L, Jamieson P, Morgan C, Murray F, Tindal A, Cruz JI, Arribas L, Fichaux N. Wind Energy - The Facts. Part I: Technology. 2nd edn., European Wind Energy Association (EWEA), 2009.

9. Bossanyi EA, Ramtharan G, Savini B. The importance of control in wind turbine design and loading. 17th Mediterranean Conference on Control and Automation, 2009; 1269-1274.

10. International Electrotechnical Commision. International Standard IEC 61400-1, Wind turbines - Part 1: Design requirements. 3rd edn., Geneva, Switzerland, 2005.

11. Burton T, Sharpe D, Jenkins N, Bossanyi E. Wind Energy Handbook. 2nd edn., John Wiley \& Sons: Chichester, UK, 2011.

12. Bryson AE, Ho YC. Applied Optimal Control. Hemisphere, Washington DC, 1975.

13. Goh CJ, Teo KL. Control parameterization: A unified approach to optimal control problems with general constraints. Automatica 1988; 24:3-18.

14. Vassiliadis VS, Sargent RWH, Pantelides CC. Solution of a class of multistage dynamic optimization problems-2. Problems with path constraints. Ind. Eng. Chem. Res. 1994; 33(9):2123-2133.

15. Biegler LT, Cervantes AM, Wächter A. Advances in simultaneous strategies for dynamic process optimization. Chem. Eng. Sci. 2002; 57(4):575-593.

16. Leineweber DB, Bauer I, Bock HG, Schlöder JP. An efficient multiple shooting based reduced SQP strategy for large-scale dynamic process optimization. Part I: Theoretical aspects. Comput. Chem. Eng. 2003; 27:157-166.

17. Srinivasan B, Bonvin D, Visser E, Palanki S. Dynamic optimization of batch processes - II: Role of measurements in handling uncertainty. Comput. Chem. Eng. 2003; 27:27-44.

18. Engell S. Feedback control for optimal process operation. J. Proc. Control 2007; 17:203-219.

19. Diehl M, Bock HG, Schlöder JP, Findeisen R, Nagy Z, Allgöwer F. Real-time optimization and nonlinear model predictive control of processes governed by differential-algebraic equations. J. Proc. Control 2002; 12(4):577-585.

20. Kadam JV, Marquardt W. Sensitivity-based solution updates in closed-loop dynamic optimization. 7th International Symposium on Dynamics and Control of Process Systems (DYCOPS), 2004.

21. Diehl M, Bock HG, Schlöder JP. A real-time iteration scheme for nonlinear optimization in optimal feedback control. SIAM J. Control Optim. 2005; 43:1714-1736.

22. Zavala VM, Laird CD, Biegler LT. Fast implementations and rigorous models: Can both be accommodated in NMPC? Int. J. Robust Nonlin. Control 2008; 18(8):800-815.

23. Gros S, Chachuat B, Bonvin D. Neighboring-Extremal Control for Singular Dynamic Optimization Problems. IIMultiple-Input Systems. Int. J. Control 2009; 82(7):1193-1211.

24. Grieder P, Kvasnica M, Baotic M, Morari M. Stabilizing low complexity feedback control of constrained piecewise affine systems. Automatica 2005; 41(10):1683-1694.

25. Pistikopoulos EN. Perspectives in multiparametric programming and explicit model predictive control. AIChE J. 2009; 55(8):1918-1925.

26. Bossanyi EA. GH Bladed, Theory Manual. 282/BR/009, Garad Hassan, 2009.

27. International Energy Agency. International recommended practices for wind energy conversion systems testing: 3 . Fatigue characteristics 1984

28. Sutherland HJ. On the Fatigue Analysis of Wind Turbines. SAND99-0089, Sandia National Laboratories, June 1999. 
29. Ben-Tal, A and El Ghaoui, L and Nemirovski, A. Robust Optimization. Series in Applied Mathematics, Princeton University Press, 2009.

30. Barton PI, Lee CK. Modeling, simulation, sensitivity analysis and optimization of hybrid systems. ACM Trans. Mod. Comput. Sim. 2002; 12(4):256-289.

31. Biegler LT. Solution of dynamic optimization problems by successive quadratic programming and orthogonal collocation. Comput. Chem. Eng. 1984; 8(3-4):243-247.

32. Gill PE, Murray W, Saunders MA. SNOPT: An SQP algorithm for large-scale constrained optimization. SIAM Rev. 2005; 47(1):99-131.

33. Bell DJ, Jacobson DH. Singular Optimal Control Problems. Academic Press, 1975.

34. Bock HG, Diehl M, Kostina E, Schlöder JP. Constrained optimal feedback control of systems governed by large differential-algebraic equations. Real-Time PDE-Constrained Optimization, Biegler L, Ghattas O, Heinkenschloss M, Keyes D, Van Bloemen Waanders B (eds.), SIAM, 2006; 3-24.

35. Würth L, Hannemann R, Marquardt W. A two-layer architecture for economically optimal process control and operation. J. Proc. Control 2011; 21(3):311-321.

36. Ferreau HJ, Bock HG, Diehl M. An online active set strategy to overcome the limitations of explicit MPC. Int. J. Robust Nonlinear Control 2008; 18(8):816-830.

37. Houska B, Ferreau M H Jand Diehl. An auto-generated real-time iteration algorithm for nonlinear MPC in the microsecond range. Automatica 2011; 47:2279-2285.

38. Gros S, Zanon M, Vukov M, Diehl M. Nonlinear MPC and MHE for mechanical multi-body systems with application to fast tethered airplanes. IFAC Conference on Nonlinear Model Predictive Control (NMPC'12), 2012.

39. Rao CV, Rawlings JB. Constrained process monitoring: Moving-horizon approach. AIChE J. 2002; 48:97-109.

40. Haverbeke N. Efficient numerical methods for moving horizon estimation. PhD Thesis, K.U. Leuven, Leuven, Belgium 2011.

41. Bottasso CL, Croce A. Cascading kalman observers of structural flexible and wind states for wind turbine control. Technical Report DIA-SR 09-02 2009; .

42. Mcgrath EL, Ridgway RW. Method for shut down of a wind turbine having rotor blades with fail-safe air brakes 2012. US Patent application number 20120134803.

43. Hameed Z, Hong YS, Cho YM, Ahn SH, Song CK. Condition monitoring and fault detection of wind turbines and related algorithms: A review. Renewable and Sustainable Energy Reviews 2009; 13(1):1-39.

44. Jonkman JM, Buhl Jr ML. FAST User's Guide. NREL/TP-500-38230, National Renewable Energy Laboratory (NREL), Golden, CO, 2005.

\section{A. FATIGUE ESTIMATION BASED ON RAIN-FLOW COUNTING}

Structural fatigue has been studied extensively in the literature and a well-accepted estimation technique is known as the rain-flow counting method (RFC) [27, 28, 11]. A typical load pattern during an EST procedure is depicted in Figure 15, with the maxima and minima in this load pattern denoted as $T_{k}$ and $C_{k}$, respectively. The RFC proceeds as follows: ${ }^{\|}$

i. The first compressive peak $C_{0}$ yields a half load cycle of magnitude $T_{0}-C_{0}$. The corresponding fatigue contribution is given by the ratio between the number of cycles performed at stress $T_{0}-C_{0}$ and the number of cycles to rupture at that stress. According to Miner's rule, the latter can be estimated as $N_{0}=c\left(T_{0}-C_{0}\right)^{-S N}$, where $S N$ and $c$ are the constants associated to the $\mathrm{S}-\mathrm{N}$ curve that models the stress-cycles-to-rupture relationship for the tower material. Therefore, the fatigue contribution is given by $\frac{1}{2 c}\left(T_{0}-C_{0}\right)^{S N}$.

ii. The first compressive peak $C_{0}$ yields another half load cycle of magnitude $T_{\infty}-C_{0}$, where $T_{\infty}$ stands for the tower load at $t \rightarrow \infty$, i.e. once the EST transient has been dampened. Following the same lines as in (i), the corresponding fatigue contribution is $\frac{1}{2 c}\left(T_{\infty}-C_{0}\right)^{S N}$.

iii. Likewise, each compressive/tensile cycles $T_{k} \rightarrow C_{k}$, with $k \geq 1$, yields a full load cycle of magnitude $T_{k}-C_{k}$, whose fatigue contribution is $\frac{1}{c}\left(T_{k}-C_{k}\right)^{S N}$.

By piecing together the various contributions, the estimated fatigue caused by the EST load pattern is given by

$$
D=\frac{1}{2 c}\left(T_{0}-C_{0}\right)^{S N}+\frac{1}{2 c}\left(T_{\infty}-C_{0}\right)^{S N}+\frac{1}{c} \sum_{k=1}^{\infty}\left(T_{k}-C_{k}\right)^{S N} .
$$

${ }^{\|}$The terminology compressive/tensile is used here as a convention. 


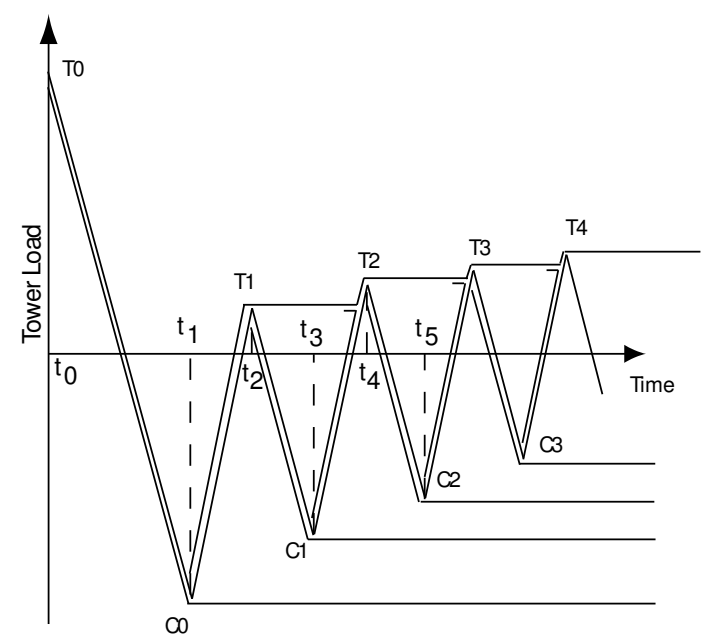

Figure 15. Rain-flow counting schematic for a typical EST load.

Under the assumption that the load peaks occur at times $t_{k}, k=1,2, \ldots$, such that $\dot{\Phi}_{T}\left(t_{k}\right) \approx 0$, and assuming that the tower load is proportional to the tower deformation $\Phi_{T}$, i.e. $T_{k}=K \Phi\left(t_{2 k}\right)$ and $C_{k}=K \Phi\left(t_{2 k+1}\right)$ for some factor $K$, the terms in the summation (25) are given by:

$$
\begin{aligned}
& T_{1}-C_{1}=K\left(\Phi_{T}\left(t_{0}\right)-\Phi_{T}\left(t_{1}\right)\right)=K \int_{0}^{t_{1}}\left|\dot{\Phi}_{T}(t)\right| d t \\
& T_{\infty}-C_{1}=K\left(\Phi_{T}(t \rightarrow \infty)-\Phi_{T}\left(t_{1}\right)\right)=K\left|\int_{t_{1}}^{\infty} \dot{\Phi}_{T}(t) d t\right| \\
& T_{k}-C_{k}=K\left(\Phi_{T}\left(t_{2 k}\right)-\Phi_{T}\left(t_{2 k+1}\right)\right)=K \int_{t_{2 k}}^{t_{2 k+1}}\left|\dot{\Phi}_{T}(t)\right| d t, \quad k=1,2, \ldots
\end{aligned}
$$

The functional (25) can therefore be rewritten in integral form as

$$
\tilde{D}=\frac{1}{2}\left(\int_{0}^{t_{1}}\left|\dot{\Phi}_{T}(t)\right| d t\right)^{S N}+\frac{1}{2}\left|\int_{t_{1}}^{\infty} \dot{\Phi}_{T}(t) d t\right|^{S N}+\sum_{k=1}^{\infty}\left(\int_{t_{2 k}}^{t_{2 k+1}}\left|\dot{\Phi}_{T}(t)\right| d t\right)^{S N}
$$

where the constants $K$ and $c$ have been omitted.

In practice, the parameter $S N$ has a typical value of 3-4, so the first few compressive/tensile cycles contribute by far the largest share in $D$, while many small cycles can be accounted for before a significant penalty is incurred. Incidentally, a large number of cycles implies an extended time horizon, which is computationally demanding.

In order to make the problem amenable to a numerical solution, the optimization horizon is taken finite as $\left[0, t_{\mathrm{f}}\right]$, and the following simple criterion is considered to approximate the structural fatigue expression (26),

$$
\tilde{D} \approx \int_{0}^{t_{\mathrm{f}}}\left|\dot{\Phi}_{T}(t)\right| d t
$$

Making this approximation is equivalent to penalizing the sum of the peak-to-peak distances in RFC. Incidentally, it also turns out to be equivalent to (26) for $S N=1$, since the following equalities hold

$$
\begin{aligned}
\tilde{D} & =\frac{1}{2} \int_{0}^{t_{1}}\left|\dot{\Phi}_{T}(t)\right| d t+\frac{1}{2}|\sum_{k=1}^{\infty} \int_{t_{2 k-1}}^{t_{2 k}} \underbrace{\dot{\Phi}_{T}(t)}_{\geq 0} d t+\sum_{k=1}^{\infty} \int_{t_{2 k}}^{t_{2 k+1}} \underbrace{\dot{\Phi}_{T}(t)}_{\leq 0} d t|+\sum_{k=1}^{\infty} \int_{t_{2 k}}^{t_{2 k+1}}\left|\dot{\Phi}_{T}(t)\right| d t \\
& =\frac{1}{2} \int_{0}^{t_{1}}\left|\dot{\Phi}_{T}(t)\right| d t+\frac{1}{2} \sum_{k=1}^{\infty} \int_{t_{2 k-1}}^{t_{2 k}}\left|\dot{\Phi}_{T}(t)\right| d t-\frac{1}{2} \sum_{k=1}^{\infty} \int_{t_{2 k}}^{t_{2 k+1}}\left|\dot{\Phi}_{T}(t)\right| d t+\sum_{k=1}^{\infty} \int_{t_{2 k}}^{t_{2 k+1}}\left|\dot{\Phi}_{T}(t)\right| d t \\
& =\int_{0}^{\infty}\left|\dot{\Phi}_{T}(t)\right| d t .
\end{aligned}
$$

